\title{
Synchrocurvature modelling of the multifrequency non-thermal emission of pulsars
}

\author{
Diego F. Torres ${ }^{\circledR}, 1,2,3 \star$ Daniele Viganò ${ }^{\circledR}, 3,4,5$ Francesco Coti Zelati ${ }^{1,2}$ and Jian $\mathrm{Li}^{6}$ \\ ${ }^{1}$ Institució Catalana de Recerca i Estudis Avançats (ICREA), E-08010 Barcelona, Spain \\ ${ }^{2}$ Institute of Space Sciences (ICE, CSIC), Campus UAB, Carrer de Can Magrans s/n, E-08193 Barcelona, Spain \\ ${ }^{3}$ Institut d'Estudis Espacials de Catalunya (IEEC), Gran Capità 2-4, E-08034 Barcelona, Spain \\ ${ }^{4}$ Departament de Física, Universitat de les Illes Balears, Palma de Mallorca, E-07122 Baleares, Spain \\ ${ }^{5}$ Institut Aplicacions Computationals (IAC3) Universitat de les Illes Balears, Palma de Mallorca, Baleares E-07122, Spain \\ ${ }^{6}$ Deutsches Elektronen Synchrotron DESY, D-15738 Zeuthen, Germany
}

Accepted 2019 August 12. Received 2019 June 17; in original form 2019 June 17

\begin{abstract}
We apply a synchrocurvature spectral emission model based on characterizing the dynamics of magnetospheric particles to fit the phase-average spectra of the most extended data base for the non-thermal spectra of pulsars. We consider 36 pulsars with well-determined non-thermal spectra from X-rays to gamma-rays. The sample includes Crab and the Crab twin, for which the spectra extend even to the optical/ultraviolet and infrared energies. We find that the model - with just three physical parameters and a global scaling - can fit the observations well across eight orders of magnitude for 18 of the 36 pulsars studied. Additionally, we find a set of eight pulsars for which the model still provides arguably good fits and another set of 10 pulsars for which the model fails in reproducing the spectra. We discuss why, propose and provide physical interpretations for a simple model extension (related to the geometry of the accelerating system with regards to the observer) that allows dealing with all such cases, ultimately providing very good fits for all pulsars. The extended model is still austere, adding only two additional parameters to the former set, of the same kind of the ones previously used. We use these fits to discuss issues going from the observed spectral origin, to the extent of the dominance of synchrotron or curvature regimes, the use of a model as predictor for searching new non-thermal pulsars starting from gamma-ray surveys, and how the model offers a setting where phase shifts between X-ray and gamma-ray light curves would naturally arise.
\end{abstract}

Key words: radiation mechanisms: non-thermal-stars: neutron.

\section{INTRODUCTION}

Coti Zelati et al. (2019) compiled a sample (complete up to 2019 April) of the non-thermal X-ray spectral energy distributions (SEDs) of the gamma-ray pulsars reported in the Fermi Second Pulsar Catalogue (Abdo et al. 2013), significantly enlarging the data availability. We now have 40 pulsars for which detailed (nonthermal) SEDs covering at least from X-rays to gamma-rays are available. Here, we embark into trying to model all such data in conjunction with the corresponding detections at higher energies.

We shall make use of the synchrocurvature, multiwavelength model that we have recently introduced by Torres (2018). We refer the reader to the Methods and supplementary materials of Torres (2018) for details, formulae, and the applicable numerical implementation, beyond what is explained below. This model is

\footnotetext{
^E-mail: dtorres@ice.csic.es
}

based on the theoretical works on synchrocurvature radiation by Viganò et al. (2015a) (see also Cheng \& Zhang 1996), and extends on the applications earlier done to study the gamma-ray data of the pulsars detected by Fermi-LAT (Viganò \& Torres 2015; Viganò et al. 2015b,c; Viganò, Torres \& Martín 2015d).

In this scheme, the emission from a pulsar occurs in one (or two, see the discussion below) accelerating region in the magnetosphere. A parallel electric field $E_{\|}$is associated to such region. The region extends from an inner $\left(x_{\mathrm{in}}\right.$, assumed to be $0.5 R_{\mathrm{lc}}$, with $R_{\mathrm{lc}}$ representing the light cylinder of the pulsar in question, $R_{\mathrm{lc}}=$ $c P / 2 \pi)$ to an outer boundary $x_{\text {out }}$, which could in principle exceed the light cylinder itself and is here taken as $1.5 R_{l c}$. It was earlier shown that a precise location and extent of the accelerating region is not dominating the predicted high-energy spectral shape of the model, justifying fixing these values for all pulsars. The accelerating region is threaded by a magnetic field assumed to be represented by a power law $B(x) \propto x^{-b}$ (see the discussion in Viganò et al. 2015c), where $x$ is the distance along the field line. The parameter 
$b$ is referred to as the magnetic gradient, and will describe how fast the magnetic field intensity declines along the trajectory of the particles. Having defined these two parameters $\left(E_{\|}, b\right)$, for a given particular pulsar - as described by its period and period derivative $(P, \dot{P})$ - the model solves the equations of motion of the particle while moving in the accelerating region, assuming they are injected or enter the region at $x_{\text {in }}$ with a (sizeable) pitch angle $\alpha$ (see Torres 2018 for the explicit formulae of the equations of motion).

The equations of motion for a single particle balance the acceleration and the radiative losses, and are used to compute physical magnitudes such as the pitch angle $\alpha$, the Lorentz factor $\Gamma$, the momentum, etc., as a function of time (or position along the trajectory). Thus, for each position of the trajectory, the physical properties under which radiation is emitted are well-determined. The radiative losses are computed taking these properties into account and considering the full synchrocurvature formulae, encompassing the transition of synchrotron-dominated emission at the initial part of the trajectory, associated with the loss of angular momentum, to a more curvature-dominated emission towards the end, when particles slide along the magnetic field lines.

Once the radiation emitted by a single particle along the trajectory across the accelerating region is known, the total yield by synchrocurvature radiation, which is what we ultimately fit to the observational data, is obtained by convolving $\mathrm{d} P_{\mathrm{sc}} / \mathrm{d} E_{\gamma}$, the singleparticle synchrocurvature spectrum (Cheng \& Zhang 1996; Viganò et al. 2015a), with the effective particle distribution, i.e. with the number of particles per unit of distance that are emitting radiation towards us, $\mathrm{d} N_{e} / \mathrm{d} x$. The explicit formula we use to fit observational data is then

$\frac{\mathrm{d} P_{\text {gap }}}{\mathrm{d} E_{\gamma}}=\int_{x_{\text {in }}}^{x_{\text {out }}}\left[\frac{\mathrm{d} P_{\text {sc }}}{\mathrm{d} E_{\gamma}}\right]\left[\frac{\mathrm{d} N_{e}}{\mathrm{~d} x}\right] \mathrm{d} x$.

Since we lack knowledge of $\mathrm{d} N_{e} / \mathrm{d} x$, we can only parametrize it. Following Torres (2018), we use the particle distribution to define (using a length scale $\left.x_{0}\right)$ the contrast, the inverse of $x_{0} /\left(x_{\mathrm{out}}-x_{\mathrm{in}}\right)$, as a measure of how uniform is the distribution of particles emitting towards us:

$\frac{\mathrm{d} N_{e}}{\mathrm{~d} x}=\left[\frac{N_{0}}{x_{0}\left(1-e^{\left.-\left(x_{\text {out }}-x_{\text {in }}\right) / x_{0}\right)}\right.}\right] e^{-\left(x-x_{\text {in }}\right) / x_{0}}$,

where $N_{0}$ is the normalization, such that $\int_{x_{\mathrm{in}}}^{x_{\text {out }}}\left(\mathrm{d} N_{e} / \mathrm{d} x\right) \mathrm{d} x=N_{0}$; its value will not affect the shape of the spectrum but only its absolute scaling. For simplicity, and in order to avoid adding degrees of freedom, we assume that all particles are created at the same location and with the same initial pitch angle. As for other parameters, these values should be taken as effective, representative of the averaged magnitudes. We come back to considerations affecting the assumed form of $\mathrm{d} N_{\mathrm{e}} / \mathrm{d} x$ below.

The single-particle synchrocurvature power, $\mathrm{d} P_{\mathrm{sc}} / \mathrm{d} E_{\gamma}$ has a cumbersome expression that is simplified by the use of the synchrocurvature parameter, $\xi$, making the power expression below (equation 3 ) to reduce itself to purely synchrotron (when $\xi \gg 1$ ) or curvature radiation (when $\xi \ll 1$ ), (Viganò et al. 2015a). It can be numerically computed as:

$\frac{\mathrm{d} P_{\mathrm{sc}}}{\mathrm{d} E}=\frac{\sqrt{3} e^{2} \Gamma y}{4 \pi \hbar r_{\mathrm{eff}}}\left[(1+z) F(y)-(1-z) K_{2 / 3}(y)\right]$,

where

$z=\left(Q_{2} r_{\mathrm{eff}}\right)^{-2}$,

$F(y)=\int_{y}^{\infty} K_{5 / 3}\left(y^{\prime}\right) \mathrm{d} y^{\prime}$,
$y=\frac{E}{E_{c}}$,

$E_{c}=\frac{3}{2} \hbar c Q_{2} \Gamma^{3}$

$r_{\mathrm{eff}}=\frac{r_{c}}{\cos ^{2} \alpha}\left(1+\xi+\frac{r_{\mathrm{gyr}}}{r_{c}}\right)^{-1}$,

$r_{\mathrm{gyr}}=\frac{m c^{2} \Gamma \sin \alpha}{e B}$,

$Q_{2}=\frac{\cos ^{2} \alpha}{r_{c}} \sqrt{1+3 \xi+\xi^{2}+\frac{r_{\mathrm{gyr}}}{r_{c}}}$

$\xi=\frac{r_{c}}{r_{\mathrm{gyr}}} \frac{\sin ^{2} \alpha}{\cos ^{2} \alpha}$.

Here, $\hbar$ is the reduced Planck constant, $c$ is the speed of light, $K_{n}$ are the modified Bessel functions of the second kind of index $n, m$ and $e$ are the rest mass and charge of the lepton, and $\alpha, r_{\mathrm{gyr}}$ and $r_{c}$ are the pitch angle, the Larmor radius, and the radius of curvature of its trajectory, respectively. $E$ is the photon energy, and $E_{c}$ is the characteristic energy of the emitted radiation. Finally, $B$ is the local strength of the magnetic field at each position. We take the following effective parametrization using the magnetic gradient:

$B(x)=B_{s}\left(R_{\star} / x\right)^{b}$,

with $B_{s}=6.4 \times 10^{19}(P[\mathrm{~s}] \dot{P})^{1 / 2} \mathrm{G}$, with $R_{\star}$ being the radius and $B_{s}$ being an estimate of the surface magnetic field of the neutron star. If particles would go in trajectories following radial lines off a dipole, $b$ would be equal to 3 . In realistic cases, we could expect a wider range of values for $b$, due to different magnetic field geometries: smaller than 3 for movements along twisted monopole or dipole lines, or larger than 3 for higher multipoles or turbulent configurations. Here, we keep it general, with the parametric formula above.

The shape of a predicted SED for a given pulsar having timing parameters $(P, \dot{P})$ is then fully determined by defining the set $\left(E_{\|}\right.$, $\left.b, x_{0} / R_{l c}\right)$, and its absolute scale by $N_{0}$. Fittings to observational data sets can then proceed by varying the former three parameters, and, for each set of them, searching the value of $N_{0}$ that minimizes the deviation between the model and the data. Further details about the fitting process (used to quantify the relative goodness-of-fit in each case) can be found in the Methods section of Torres (2018). It is interesting to note that the model can then be used to show the contribution of each part of the particles trajectories to the total predicted yield at each band, as we will show below.

It is also relevant to note that $x_{0} / R_{l c}$ and $N_{0}$ are parameters holding relation with the geometry of the system. For instance, the number of particles emitting radiation directed towards the observer can be different from two accelerating regions located at different positions in the magnetosphere, despite their physical parameters $\left(E_{\|}, b\right)$ being potentially the same (e.g. if we assume symmetric accelerating regions in the two hemispheres, with different Earth's visibility of particle directions). We come back to this below.

Despite the relative simplicity of the conceptual approach of this model and the reduced number of the physical parameters that are to be varied, it has proven successful in describing the X-ray and gamma-ray emission of several known pulsars (see the cases shown 

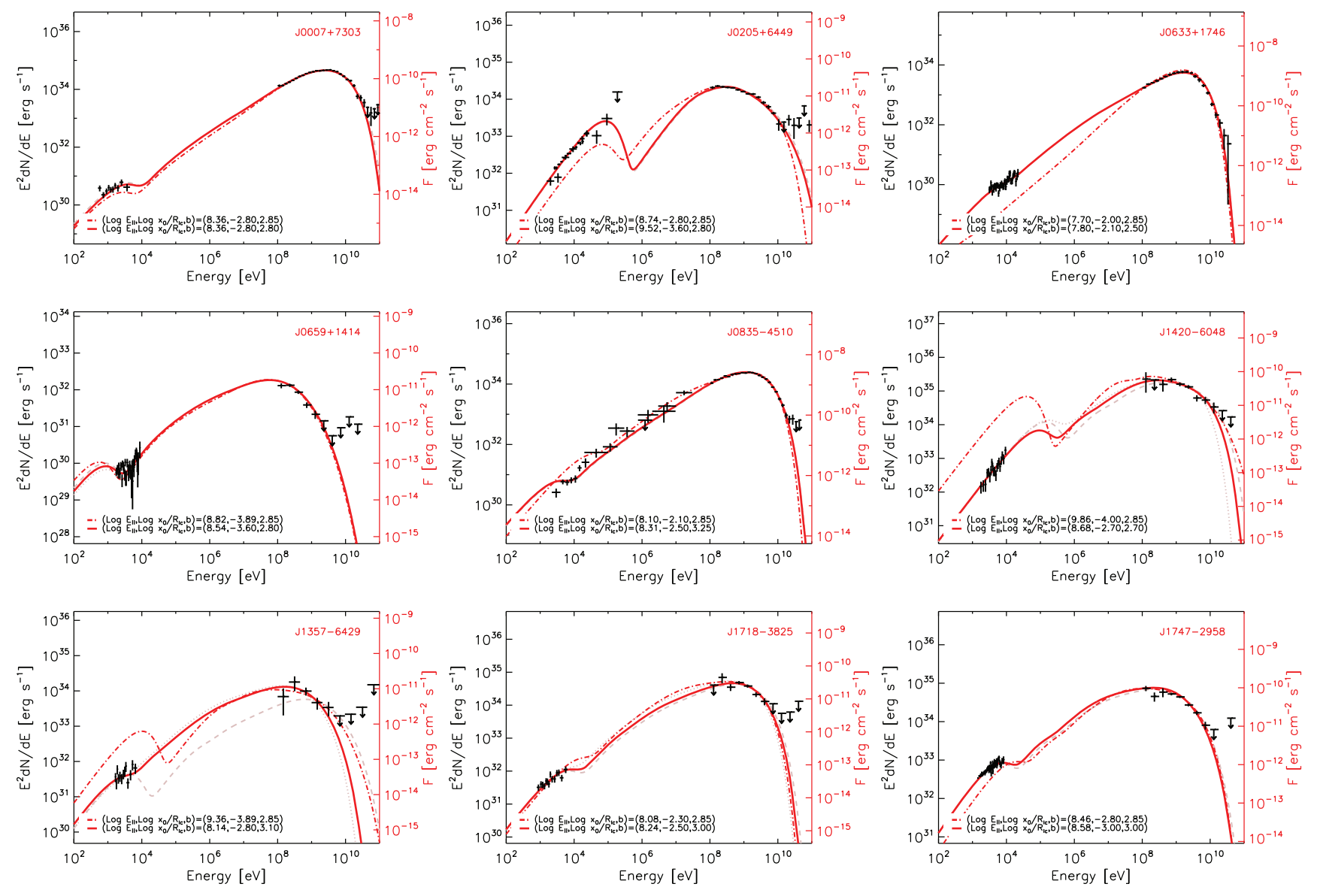

Figure 1. Broad-band SEDs well-describable with a single set of $\left(E_{\|}, b, x_{0} / R_{l c}, N_{0}\right)$ parameters. The panels show the corresponding theoretical model fits to the broad-band SEDs of several pulsars using a single set of parameters $\left(E_{\|}, b, x_{0} / R_{l c}, N_{0}\right)$ as given in the legend. See the first panel of Table 1 for the uncertainties of the best-fitting parameters. The solid red line is the best-fit of our model to the full observational data set (black crosses indicating detections with their statistical $1 \sigma$ errors, and black arrows marking upper limits). The two milder, grey-coloured curves lying close to the solid line correspond to the predicted SEDs resulting from varying $E_{\|}$and $x_{0}$ within their maximum ranges of their $1 \sigma$ uncertainty. The red dash-dotted lines correspond to the best-fit SEDs obtained when considering the gamma-ray data only. See the text for further details.

in Torres 2018). The model was also used to predict pulsars that should be detectable in X-rays out of their detected gamma-ray emission ( $\mathrm{Li}$ et al. 2018; Torres 2018). Here, we shall confront this model to a larger number of broad-band observational data sets, recalling that prior to the observational analysis effort described in Coti Zelati et al. (2019), only a few X-ray to gamma-ray SEDs were available. In what follows, then, we apply this spectral model to the whole sample of broad-band SEDs for PSRs and MSPs we have earlier compiled.

\section{MODELLING NON-THERMAL SEDS}

The sample of certainly non-thermal, X-ray emitting, gamma-ray pulsars contains 33 pulsars (PSRs, with $P>10 \mathrm{~ms}$ ), and $7 \mathrm{~ms}$ pulsars (MSPs, with $P<10 \mathrm{~ms}$ ) (Coti Zelati et al. 2019). From this we exclude the three MSPs detailed below, requiring additional FermiLAT analysis in order to have a consistent set of data (we briefly comment on the results we obtain using these refurbished data set below) and the PSR J2043+2740, due to the current scarcity of data points in its SED (it has only seven data points useful for our fitting summing up the X-ray and gamma-ray domain). In total, then, we shall consider 32 PSRs and 4 MSPs here.

\subsection{Broad-band SEDs well-describable with a single set of $\left(E_{\|}\right.$, $\left.b, x_{0} / R_{l c}, N_{0}\right)$ parameters}

We found that in 18 out of the 36 pulsars herein considered, the model with a single set of parameters $\left(E_{\|}, b, x_{0} / R_{l c}, N_{0}\right)$, as used by Torres (2018), is able to qualitatively describe well the observational data across seven to eight orders of magnitude in energy. This is explicitly shown in Fig. 1.

Each panel of Fig. 1 show several curves: the solid red line is the best-fit of our model to the full observational data set. For each case, the two milder, grey-coloured curves lying close to the solid line correspond to the predicted SEDs resulting from varying $E_{\|}$and $x_{0}$ within their maximum ranges of their $1 \sigma$ uncertainty. In particular, we plot with a dotted line the minimum (maximum) in the range of $E_{\|}\left(x_{0} / R_{\mathrm{lc}}\right)$ and the corresponding value of $b$ (usually the minimum in the range of $b$ ); and with a dashed line the maximum (minimum) in the range of $E_{\|}\left(x_{0} / R_{\mathrm{lc}}\right)$ and the corresponding value of $b$ (usually the maximum in the range of $b$ ). They give an idea of the overall uncertainty of the predicted SED. When the uncertainties in the fitted parameters are low, these curves overlap in the scale plotted.

In the same Fig. 1 we also plot the best-fitting SEDs obtained when considering the gamma-ray data only, indicated by red dashdotted lines. In these fits, we fix $b=2.85$ in all cases, in order 

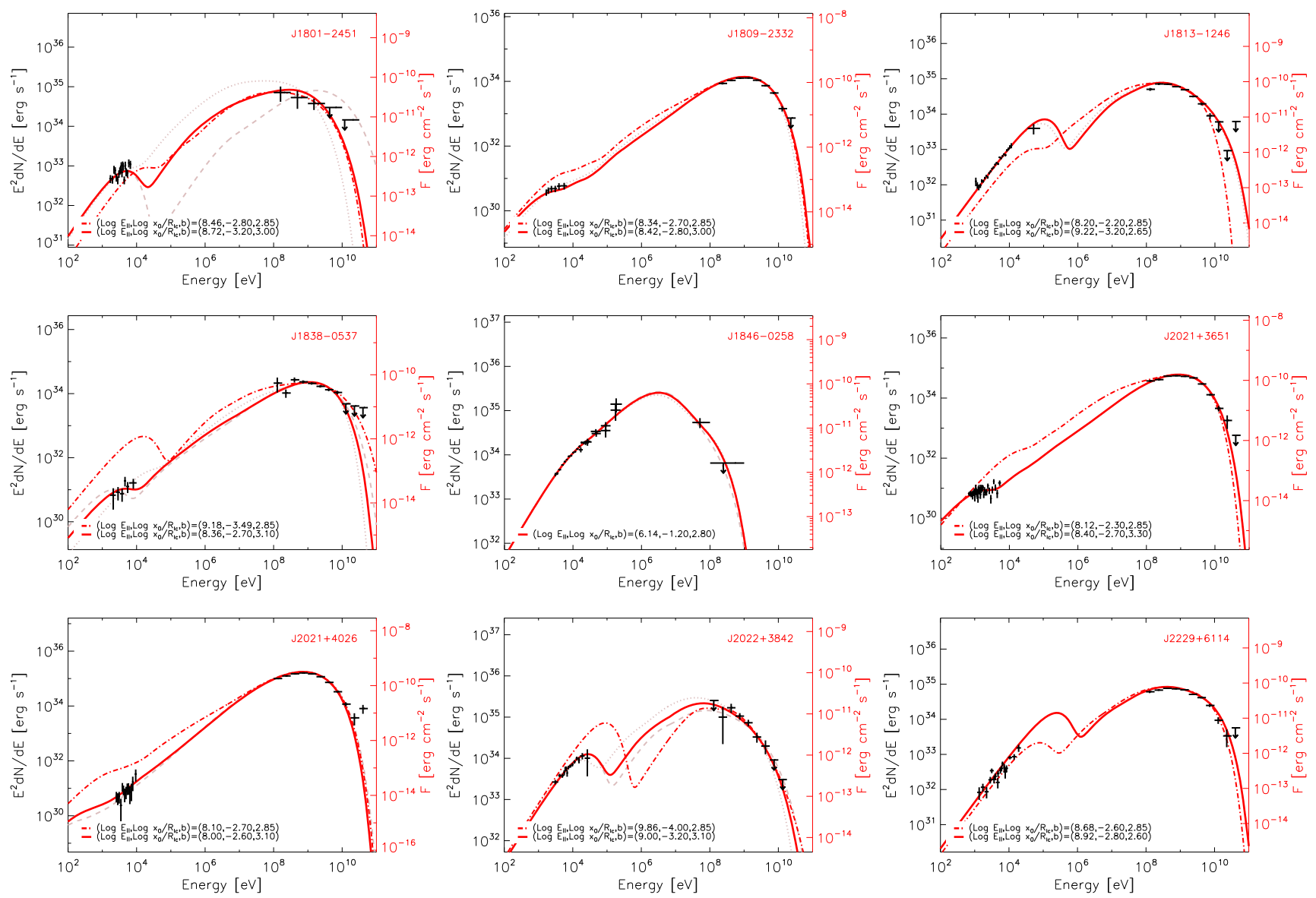

Figure 1 - continued

to reduce the degrees of freedom. This choice is motivated by the fact that, as it was shown in previous studies (see Viganò et al. 2015c; Torres 2018) the high-energy gamma-ray data can barely distinguish the value of $b$, especially if there is a lack of welldetermined points at low and high gamma-ray energies. This is also confirmed by these figures. Physically, this happens because the $b$-parameter (i.e. the magnetic gradient of the field intensity) affects mostly the synchrotron-dominated synchrocurvature radiation, whereas the gamma-ray emission occurs in full synchrocurvature, and sometimes, in curvature-dominated regimes. In the fitting to the gamma-ray data only, then, the dotted-line model of Fig. 1 is based on the exploration of only two parameters $\left(E_{\|}, x_{0}\right)$ and a global scaling (the normalization $N_{0}$ ). The central values of all the best-fitting parameters are quoted within each panel, whereas the fitted parameters and uncertainties of the X-ray to gamma-ray fit are given also in the first panel of Table 1. The latter table also shows the pulsar type, name, the distance used in the conversion of flux to luminosities, the best-fitting values, and their $1 \sigma$ uncertainty.

Fig. 1 shows that the gamma-ray-based best-fits SEDs can be used to predict the X-rays, where they result to be close (typically within a factor of a few) to the observed SED in that energy range. This extended sample confirms what was advanced by Torres (2018): using the model to fit the gamma-ray data only (available from the Fermi-LAT or other future surveys) could predict which pulsars could be observable in X-rays.

Fig. 2 provides examples of the total SED formation for some of the pulsars of our sample, dissecting how the model works. Each curve plotted in the figure represents the contribution of a given portion of the particles's trajectory along the accelerating region, whose position is colour-coded at the top. In order to make the figure legible, not all spatial bins actually computed are shown. The sum of all these contributions constitutes the final fit, depicted by the red curve (which corresponds to the fit shown in Fig. 1). Fig. 2 is then similar to fig. 2 of Torres (2018), and shows how the same mechanism applies for all the new cases studied in Fig. 1. We see that the X-ray emission is produced as a result of the emission at the initial part of the particle trajectories, when there is a large loss of the momentum component perpendicular to the magnetic field, and a consequent fast decrease of the pitch angle. In turn, the gammaray emission is produced further out in the particle's evolution, as a result of synchrocurvature emission that starts (but in most cases not yet dramatically) being dominated by curvature.

This can be seen better in conjunction with the analysis of the properties of the trajectories themselves, output of the equations of motion. Fig. 3 shows three of the most important quantities entering into the computation of the SED: the Lorentz factor, the synchrocurvature parameter (recall that $\xi=1$ formally marks the transition from synchrotron $(\xi \ll 1)$ to curvature $(\xi \gg 1)$ dominated emission) and the pitch angle. Note how synchrotron emission is very relevant in the initial and less pronounced drop of the pitch angle, and how the Lorentz factor grows linearly when synchrocurvature dominates, until it saturates when the curvature-dominated radiation is large enough to balance the electric acceleration. 
Table 1. Results of the broad-band spectral modelling with a single set of $\left(\log \left(E_{\|}\right), b, \log \left(x_{0} / R_{l c}\right), \log \left(N_{0}\right)\right)$ parameters. The table shows the pulsar type, name, distance used in the conversion of flux to luminosities, the bestfitting values, and their $1 \sigma$ uncertainty in the form $\left(\log \left(E_{\|}\right), b, \log \left(x_{0} / R_{l c}\right)\right.$, $\left.\log \left(N_{0}\right)\right)$. The horizontal lines divide the pulsars whose SEDs are well represented by a single set of parameters (Fig. 1), those that are arguably good (Fig. 5), and those for which one set of parameters is clearly not enough, suggesting we may be seeing significant contribution from two regions of acceleration or that the geometrical configuration is such that a single distribution is not valid at all energies (Fig. 6).

\begin{tabular}{|c|c|c|c|c|c|c|}
\hline Type & Pulsar & $\begin{array}{c}D \\
(\mathrm{kpc})\end{array}$ & $\begin{array}{c}\log E_{\| 1} \\
\left(\mathrm{~V} \mathrm{~m}^{-1}\right)\end{array}$ & $b$ & $\log \left(x_{0} / R_{\mathrm{lc}}\right)$ & $\log N_{0}$ \\
\hline PSR & $\mathrm{J} 0007+7303$ & 1.40 & $8.36_{-0.02}^{+0.10}$ & $2.80_{-0.10}^{+0.10}$ & $-2.80_{-0.10}^{+0.10}$ & $32.22_{-0.01}^{+0.01}$ \\
\hline PSR & $\mathrm{J} 0205+6449$ & 3.20 & $9.52_{-0.02}^{+0.02}$ & $2.80_{-0.10}^{+0.10}$ & $-3.60_{-0.10}^{+0.10}$ & $30.67_{-0.01}^{+0.01}$ \\
\hline PSR & $\mathrm{J} 0633+1746$ & 0.19 & $7.80_{-0.02}^{+0.02}$ & $2.50_{-0.10}^{+0.10}$ & $-2.10_{-0.10}^{+0.10}$ & $31.15_{-0.01}^{+0.01}$ \\
\hline PSR & $\mathrm{J} 0659+1414$ & 0.29 & $8.54_{-0.10}^{+0.10}$ & $2.80_{-0.10}^{+0.10}$ & $-3.60_{-0.10}^{+0.11}$ & $31.98_{-0.01}^{+0.01}$ \\
\hline PSR & J0835-4510 & 0.28 & $8.31_{-0.01}^{+0.01}$ & $3.25_{-0.05}^{+0.05}$ & $-2.50_{-0.10}^{+0.10}$ & $32.05_{-0.04}^{+0.01}$ \\
\hline PSR & $\mathrm{J} 1420-6048$ & 5.60 & $8.68_{-0.48}^{+0.26}$ & $2.70_{-0.10}^{+0.10}$ & $-2.70_{-0.20}^{+0.50}$ & $31.95_{-0.37}^{+0.30}$ \\
\hline PSR & J1357-6429 & 3.10 & $8.14_{-0.32}^{+0.98}$ & $3.10_{-0.10}^{+0.10}$ & $-2.80_{-0.80}^{+0.30}$ & $32.96_{-1.05}^{+0.10}$ \\
\hline PSR & J1718-3825 & 3.60 & $8.24_{-0.22}^{+0.12}$ & $3.00_{-0.10}^{+0.10}$ & $-2.50_{-0.10}^{+0.20}$ & $32.65_{-0.10}^{+0.15}$ \\
\hline PSR & J1747-2958 & 2.52 & $8.58_{-0.02}^{+0.12}$ & $3.00_{-0.10}^{+0.10}$ & $-3.00_{-0.10}^{+0.10}$ & $33.13_{-0.09}^{+0.01}$ \\
\hline PSR & J1801-2451 & 3.80 & $8.72_{-0.70}^{+1.02}$ & $3.00_{-0.10}^{+0.10}$ & $-3.20_{-0.80}^{+0.60}$ & $33.24_{-0.78}^{+0.49}$ \\
\hline PSR & J1809-2332 & 0.88 & $8.42_{-0.26}^{+0.02}$ & $3.00_{-0.10}^{+0.10}$ & $-2.80_{-0.10}^{+0.30}$ & $32.10_{-0.14}^{+0.01}$ \\
\hline PSR & J1813-1246 & 2.63 & $9.22_{-0.12}^{+0.02}$ & $2.65_{-0.05}^{+0.05}$ & $-3.20_{-0.10}^{+0.10}$ & $31.17_{-0.01}^{+0.18}$ \\
\hline PSR & J1838-0537 & 1.80 & $8.36_{-0.34}^{+0.36}$ & $3.10_{-0.30}^{+0.10}$ & $-2.70_{-0.40}^{+0.50}$ & $32.15_{-0.58}^{+0.16}$ \\
\hline PSR & J1846-0258 & 5.80 & $6.14_{-0.08}^{+0.06}$ & $2.80_{-0.10}^{+0.10}$ & $-1.20_{-0.10}^{+0.10}$ & $34.57_{-0.06}^{+0.05}$ \\
\hline PSR & $\mathrm{J} 2021+3651$ & 1.80 & $8.40_{-0.02}^{+0.02}$ & $3.30_{-0.10}^{+0.10}$ & $-2.70_{-0.10}^{+0.10}$ & $32.74_{-0.01}^{+0.01}$ \\
\hline PSR & $\mathrm{J} 2021+4026$ & 2.15 & $8.00_{-0.02}^{+0.18}$ & $3.10_{-0.10}^{+0.10}$ & $-2.60_{-0.20}^{+0.10}$ & $33.61_{-0.01}^{+0.07}$ \\
\hline PSR & $\mathrm{J} 2022+3842$ & 10.00 & $9.00_{-0.36}^{+0.24}$ & $3.10_{-0.10}^{+0.10}$ & $-3.20_{-0.20}^{+0.30}$ & $32.59_{-0.19}^{+0.53}$ \\
\hline PSR & $\mathrm{J} 2229+6114$ & 3.00 & $8.92_{-0.02}^{+0.02}$ & $2.60_{-0.10}^{+0.10}$ & $-2.80_{-0.10}^{+0.10}$ & $31.19_{-0.01}^{+0.01}$ \\
\hline PSR & $\mathrm{J} 0357+3205$ & 0.83 & $6.92_{-0.12}^{+0.08}$ & $2.20_{-0.10}^{+0.10}$ & $-1.30_{-0.20}^{+0.20}$ & $31.04_{-0.03}^{+0.17}$ \\
\hline PSR & J1513-5908 & 4.40 & $6.23_{-0.01}^{+0.01}$ & $2.75_{-0.05}^{+0.05}$ & $-1.10_{-0.10}^{+0.10}$ & $35.01_{-0.01}^{+0.01}$ \\
\hline PSR & J1709-4429 & 2.60 & $8.30_{-0.02}^{+0.02}$ & $3.10_{-0.10}^{+0.10}$ & $-2.50_{-0.10}^{+0.10}$ & $33.11_{-0.01}^{+0.01}$ \\
\hline PSR & J1826-1256 & 1.55 & $8.26_{-0.02}^{+0.02}$ & $3.10_{-0.10}^{+0.10}$ & $-2.60_{-0.10}^{+0.10}$ & $32.58_{-0.01}^{+0.01}$ \\
\hline PSR & J1833-1034 & 4.10 & $9.86_{-0.12}^{+0.12}$ & $3.30_{-0.10}^{+0.10}$ & $-4.10_{-0.12}^{+0.10}$ & $32.79_{-0.09}^{+0.08}$ \\
\hline PSR & $\mathrm{J} 1836+5925$ & 0.30 & $7.76_{-0.02}^{+0.02}$ & $2.60_{-0.10}^{+0.10}$ & $-2.00_{-0.10}^{+0.10}$ & $30.86_{-0.01}^{+0.01}$ \\
\hline PSR & $\mathrm{J} 2030+4415$ & 0.88 & $9.24_{-0.78}^{+0.10}$ & $2.90_{-0.10}^{+0.10}$ & $-3.90_{-0.10}^{+0.80}$ & $31.99_{-0.11}^{+0.01}$ \\
\hline PSR & $\mathrm{J} 2055+2539$ & 0.62 & $9.43_{-0.01}^{+0.01}$ & $2.70_{-0.10}^{+0.10}$ & $-4.10_{-0.10}^{+0.10}$ & $31.39_{-0.03}^{+0.01}$ \\
\hline PSR & $\mathrm{J} 1057-5226$ & 0.30 & $7.40_{-0.02}^{+0.02}$ & $5.00_{-0.10}^{+0.10}$ & $-1.50_{-0.10}^{+0.10}$ & $30.39_{-0.01}^{+0.01}$ \\
\hline PSR & J1124-5916 & 5.00 & $8.20_{-0.02}^{+0.02}$ & $3.20_{-0.10}^{+0.10}$ & $-2.90_{-0.10}^{+0.10}$ & $34.17_{-0.01}^{+0.01}$ \\
\hline PSR & J1741-2054 & 0.30 & $9.10_{-0.02}^{+0.02}$ & $2.65_{-0.05}^{+0.05}$ & $-3.96_{-0.10}^{+0.10}$ & $31.59_{-0.01}^{+0.01}$ \\
\hline PSR & $\mathrm{J} 1952+3252$ & 3.00 & $9.64_{-0.02}^{+0.02}$ & $3.30_{-0.10}^{+0.10}$ & $-4.00_{-0.10}^{+0.10}$ & $33.37_{-0.01}^{+0.01}$ \\
\hline MSP & J0437-4715 & 0.16 & $11.04_{-0.14}^{+0.02}$ & $2.90_{-0.10}^{+0.10}$ & $-4.52_{-0.10}^{+0.10}$ & $29.17_{-0.01}^{+0.08}$ \\
\hline MSP & J0614-3329 & 0.62 & $11.80_{-0.02}^{+0.02}$ & $4.10_{-0.10}^{+0.10}$ & $-5.00_{-0.10}^{+0.10}$ & $29.84_{-0.01}^{+0.01}$ \\
\hline MSP & $\mathrm{J} 0751+1807$ & 1.10 & $11.50_{-0.02}^{+0.02}$ & $3.30_{-0.10}^{+0.10}$ & $-4.70_{-0.10}^{+0.10}$ & $29.60_{-0.01}^{+0.02}$ \\
\hline MSP & J1231-1411 & 0.42 & $8.80_{-0.02}^{+0.02}$ & $2.50_{-0.10}^{+2.50}$ & $-1.60_{-0.10}^{+0.10}$ & $28.57_{-0.01}^{+0.20}$ \\
\hline
\end{tabular}

Examples of how the SEDs are affected by the contrast parameter are shown in Fig. 4, and again we find similarity with the cases studied earlier by Torres (2018). A large variety of spectral shapes can be obtained from the same pulsar, the same synchrocurvature process, and the same accelerating region (whose physical properties are fixed by the accelerating field and magnetic field gradient), just as a result of the variations in the contrast.

Fig. 4 already poses the issue of how difficult it may be to predict the whole SED if having access to X-ray data only. Unless the $\mathrm{X}$-ray data uncertainty is very low, and its energy coverage large, there might be a qualitative degeneracy in the contrast values in such cases. As an example, focus on the middle panel of the top row of Fig. 4, the case of PSR J1709-3825. If only the X-ray data set is available, a fit with low $x_{0} / R_{\mathrm{lc}}$ (dark curves) can be (at least qualitatively) as good as one with larger values (lighter curves). Such degeneracy can of course be broken by high-quality X-ray data, and even better, by harder X-ray or eventually by MeV data. Note however that this degeneracy is much less severe starting from gamma-ray data (i.e. gamma-rays are a better predictor for the total SED): a good sampling in this energy domain leaves little freedom to the value of $x_{0} / R_{\mathrm{lc}}$ in all the cases where a single set of parameters is already enough to represent the whole SED well.

\subsection{Additional broad-band SEDs arguably well-describable with a single set of $\left(E_{\|}, b, x_{0} / R_{l c}, N_{0}\right)$ parameters}

The panels of Fig. 5 show a second set of eight pulsars in our sample where the best-fitting models, while maintaining the general broad-band trend shown by the available data, misses a few of the observational points or a given peculiarity of the spectrum. The parameters of the common X-ray and gamma-ray fittings of Fig. 5 are given in the second panel of Table 1. In particular, we notice the following mismatches:

$J 0357+3205$ : the model is not describing well the flatness of the X-ray data set.

J1513-5908: the model seems to overpredict the $1 \mathrm{MeV}$ flux.

J1709-4429: the model does not correctly describe the peculiar $\mathrm{X}$-ray spectrum, described with data featuring small error bars.

J826-1256: the model is missing the lowest energy gamma-ray point (which, however, has a large error bar) and the highest energy $\mathrm{X}$-ray data.

J1833-1034: the model is incompatible with the upper limit of the smallest energy gamma-ray bin.

J1836-5925: the model is missing the flatness of the X-ray SED.

$J 2030+4415$ : the model is incompatible with the upper limit of the smallest energy gamma-ray bin.

$J 2055+2539$ : the model is missing the highest energy X-ray data (too flat for the best fit) and the slope of the gamma-ray spectrum.

Despite these mismatches, the overall look of all fits of Fig. 5 can be considered reasonable. This conclusion is emphasized recalling that the theoretical spectral shape only depends on three parameters and that the lowest gamma-ray point may be the most affected by the treatment of the diffuse emission and/or the removal of any pulsar wind nebula component, thus and care must be exercised if accepting or rejecting a model only based on such data point only.

However, perhaps the inability of capturing the spectral flatness in X-rays and/or the lowest or highest energy data points in X-rays or gamma-rays may indicate an over simplification of our model. We shall come back to these cases below, after considering a few pulsars for which the model is indeed undoubtedly unable to find a good SED fitting with a single set of $\left(E_{\|}, b, x_{0} / R_{l c}, N_{0}\right)$ parameters.

\subsection{Broad-band SEDs not describable with a single set of $\left(E_{||}\right.$, $\left.b, x_{0} / R_{l c}, N_{0}\right)$ parameters}

The third group on which we focus is formed by eight sources for which the use of our model with a single set of $\left(E_{\|}, b, x_{0} / R_{l c}\right.$, $N_{0}$ ) parameters indeed fails to produce a good fit (see Fig. 6). For reference, the values of these fitted parameters are also separately quoted in the final panel of Table 1 in all these cases. 

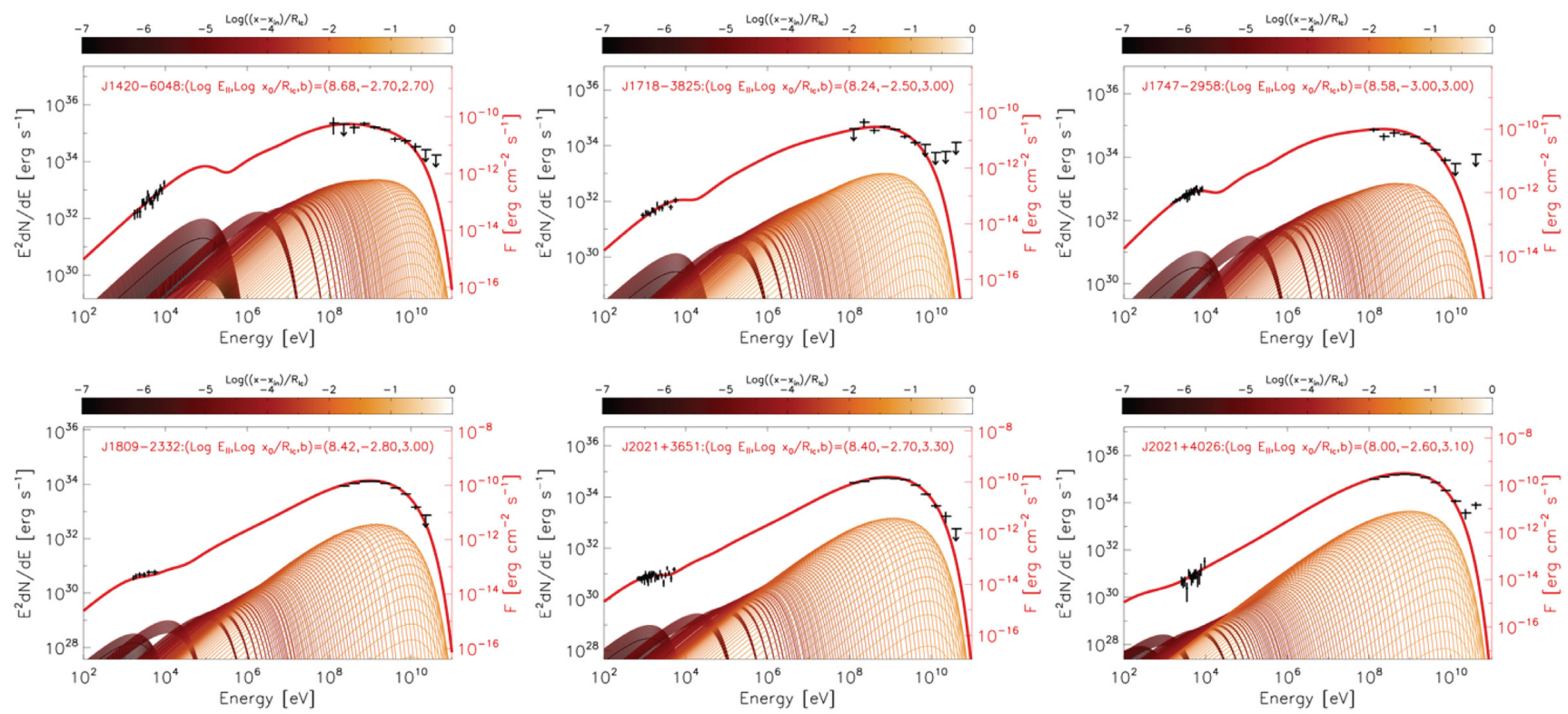

Figure 2. Contribution of each part of the particle trajectories along the accelerating regions (colour-coded at the top) to the total fit in six examples of the pulsars studied in Fig. 1. See the text for discussion.
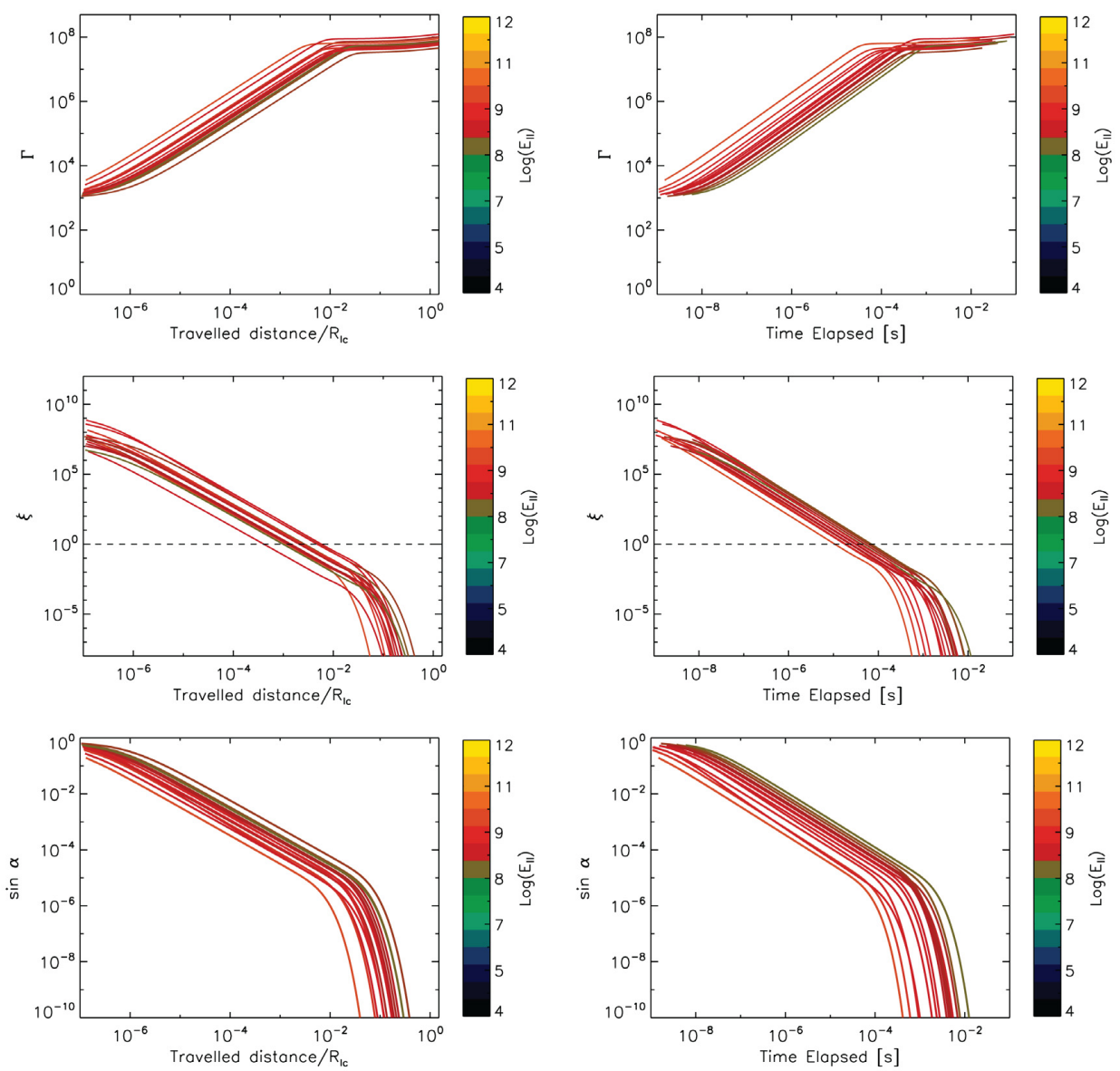

Figure 3. Main properties of the trajectories of particles that generate the SED fittings of Fig. 1, both as a function of travelled distance (left-hand panels) and time elapsed since injection (right-hand panels). The colour coding is used to represent the value of the accelerating electric field, in units of $\mathrm{V} \mathrm{m}^{-1}$. 

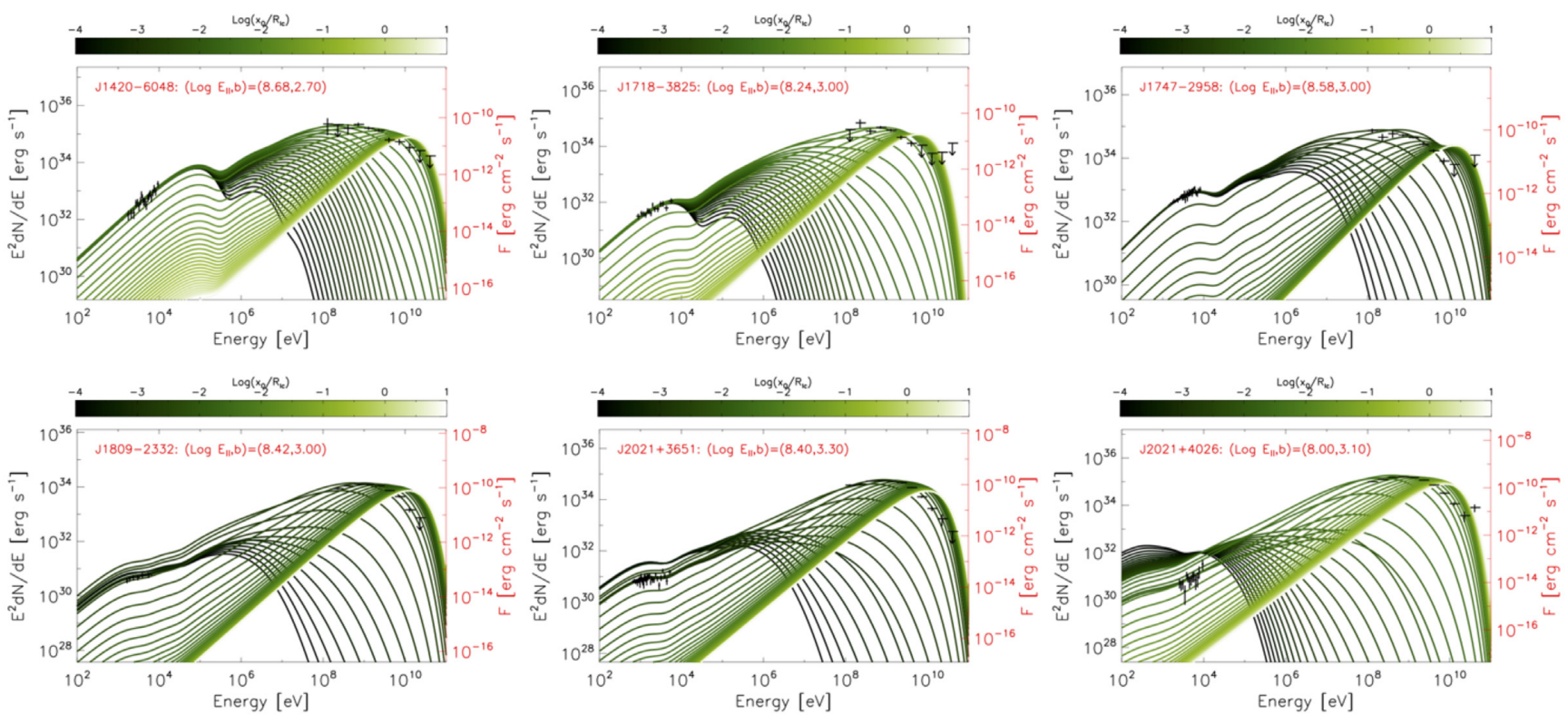

Figure 4. The same six examples of Fig. 2 used to show the influence of the contrast parameter. Each of the panels has the electric field and magnetic gradient fixed at the values found to be the best fit to the data in Fig. 1. The contrast is colour-coded from uniform (formally, $x_{0} / R_{\mathrm{lc}} \rightarrow \infty$, white colour), to finite decreasing values $x_{0} / R_{\mathrm{lc}} \in\left[10^{-1}-10^{-5}\right]$ (light to dark colour).
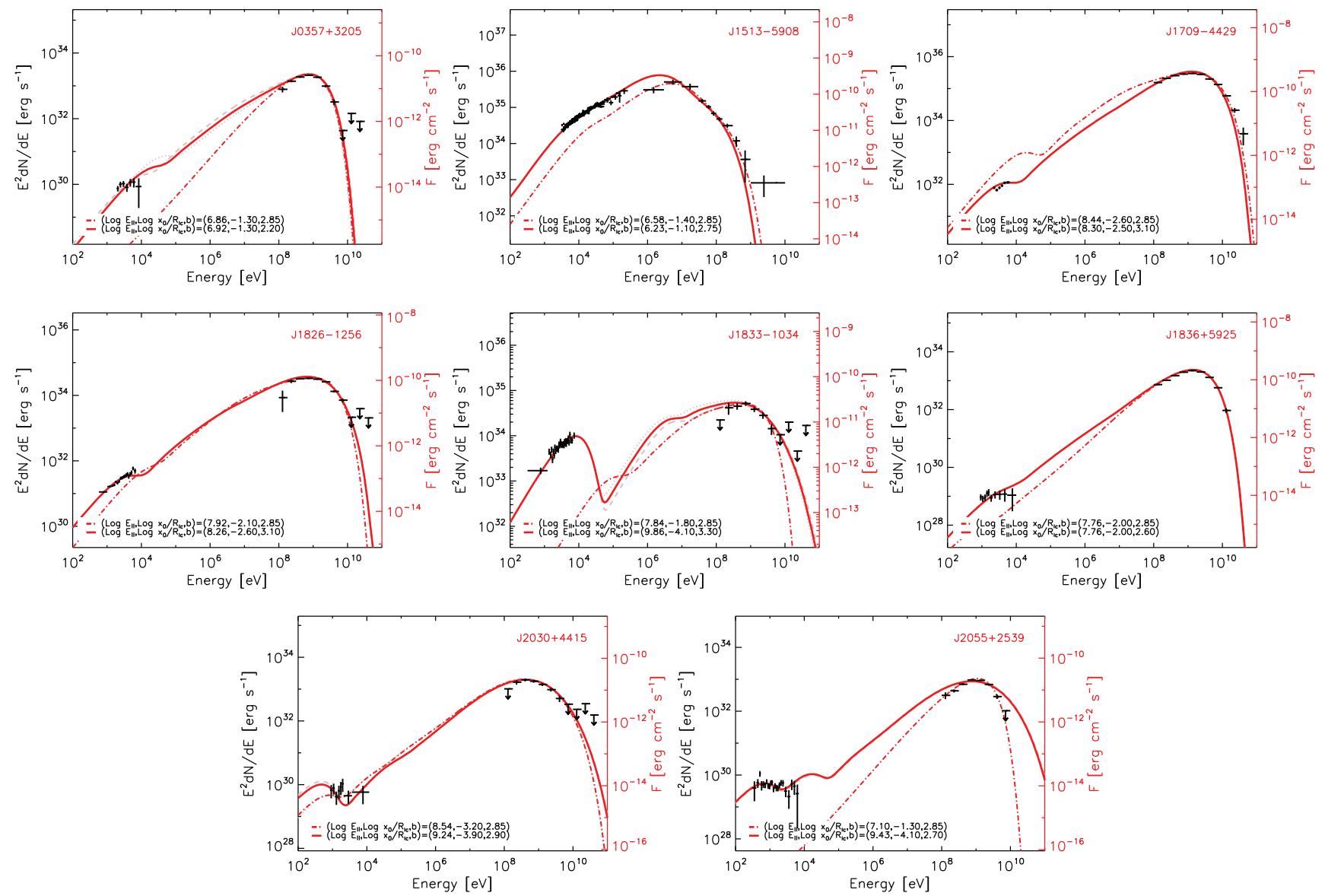

Figure 5. Broad-band SEDs arguably well-describable with a single set of $\left(E_{\|}, b, x_{0} / R_{l c}, N_{0}\right)$ parameters. The panels show the fits to the SEDs of several pulsars (according to the corresponding label) using a single set of parameters $\left(E_{\|}, b, x_{0} / R_{\mathrm{lc}}, N_{0}\right)$ in our theoretical model. In these cases, however, one can see that the model is failing in describing some specific properties of the data, see the text. Curves shown are as in Fig. 1. 

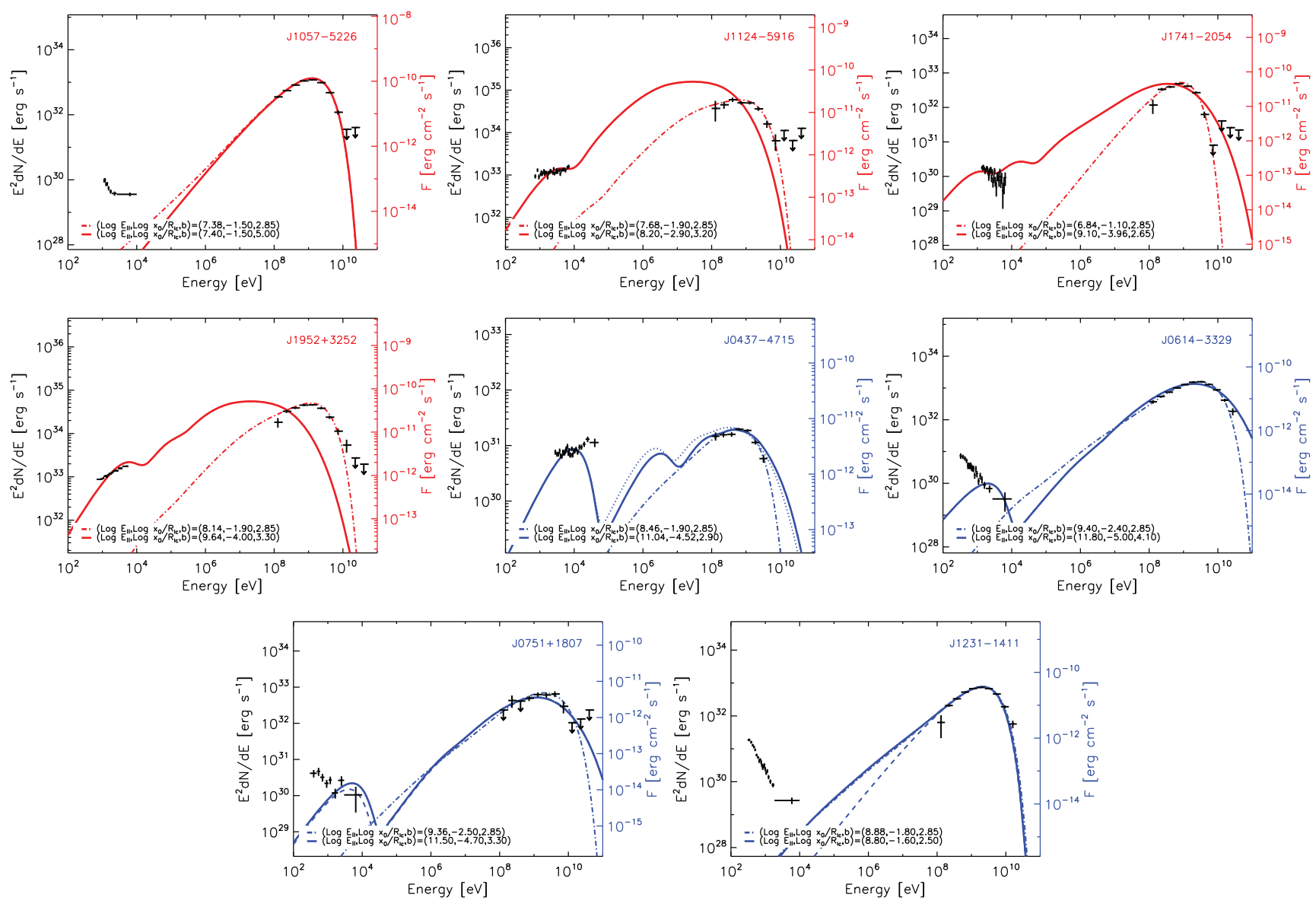

Figure 6. Broad-band SEDs not describable with a single set of $\left(E_{\|}, b, x_{0} / R_{\mathrm{lc}}, N_{0}\right)$ parameters. The panels show the SEDs of pulsars in our sample for which an acceptable fit using a single set of parameters $\left(E_{\|}, b, x_{0} / R_{\mathrm{lc}}, N_{0}\right)$ is not found. We use red for pulsars with $P>10$ ms and blue for MSPs. Curves shown are as in Fig. 1.

Why is the model unable to fit the data of these pulsars? First of all, one should recall that the parameters $(P, \dot{P})$ defining the specific pulsar are taken into account when fitting. Thus, fitting a specific SED could be possible for a specific pulsar, but maybe not for another; comparing just the similarity of the spectra does not show the whole picture.

On the other hand, some cases show an inverted X-ray spectra Coti Zelati et al. (2019), for which the X-ray SED is decreasing with energy (e.g. as PSR J1057-5227, J1741-2054, and the MSPs). This could in principle be fitted in the decaying part of the synchrotrondominated radiation.

The consideration of the X-ray data set for the pulsars in Figs 5 and 6 has the result of worsening how the model describes the gamma-ray data (see e.g. J1057-5226, J1741-2054, J1836+5925, $\mathrm{J} 2055+2539$ ), up to the point of completely missing the highenergy data points when approaching the X-ray data in turn (see e.g. J1952+3252, or J1124-5916). A similar situation occurs for the MSPs studied here. In other words, for these pulsars we find that the best-fitting model tends to minimize the deviations from observed data by approaching either the X-ray data or the gammaray data; coping with both is impossible.

To understand better how this happens we show examples of our search for a best fit in Figs 7 and 8. We take J1741-2054 as a study case, but the general scenario is similar for the other pulsars too. Each panel of Fig. 7 shows changes of $E_{\|}$for a given value of $b$ (fixed at $b=3$ in this example), varying $x_{0} / R_{\mathrm{lc}}$ from $10^{-5}$ to 1 , shown with the colour scale (blue-green-red-yellow scale, with the addition of the uniform case, $x_{0} \rightarrow \infty$, the brightest curve). At relatively low values of $E_{\|}$we find that the model can minimize the deviations from data either trying to fit the X-ray observations (at low $x_{0} / R_{\mathrm{lc}}$ values, the blue curves), or the gamma-ray observations (at larger $x_{0} / R_{\mathrm{lc}}$ values, between green and red curves). No single curve is close to both data sets.

A similar effect is shown when varying the magnetic gradient $b$ for a fixed value of $E_{\|}$. Fig. 8 shows a few such examples, using $b=$ 2.5, 3.0, and 3.5 for a fixed value of $E_{\|}$and with the same ranging of the $x_{0} / R_{\mathrm{lc}}$ values as commented above, represented in the colour coding. Depending on the relative values of $b$ and $x_{0} / R_{\mathrm{lc}}$, again the fit prefers to minimize the deviation either approaching the X-ray or the gamma-ray data, but it cannot do it for both at once either.

Figs 7 and 8 show only a set of the examples we explored. When searching for a common fit, we systematically vary $b$ and $E_{\|}$in a wide range, in addition to the exploration of $x_{0} / R_{\mathrm{lc}}$ for each case, substantiating that no good fit using a single set of parameters can be found in these cases.

We note that the inability to fit the broad-band non-thermal spectra as commented is not common to all MSPs known. It just so happens that the four analysed here are in this set, but our preliminary results using a reanalysis of the gamma-ray data for the remaining three MSPs with broad-band spectra, B1821-24, 

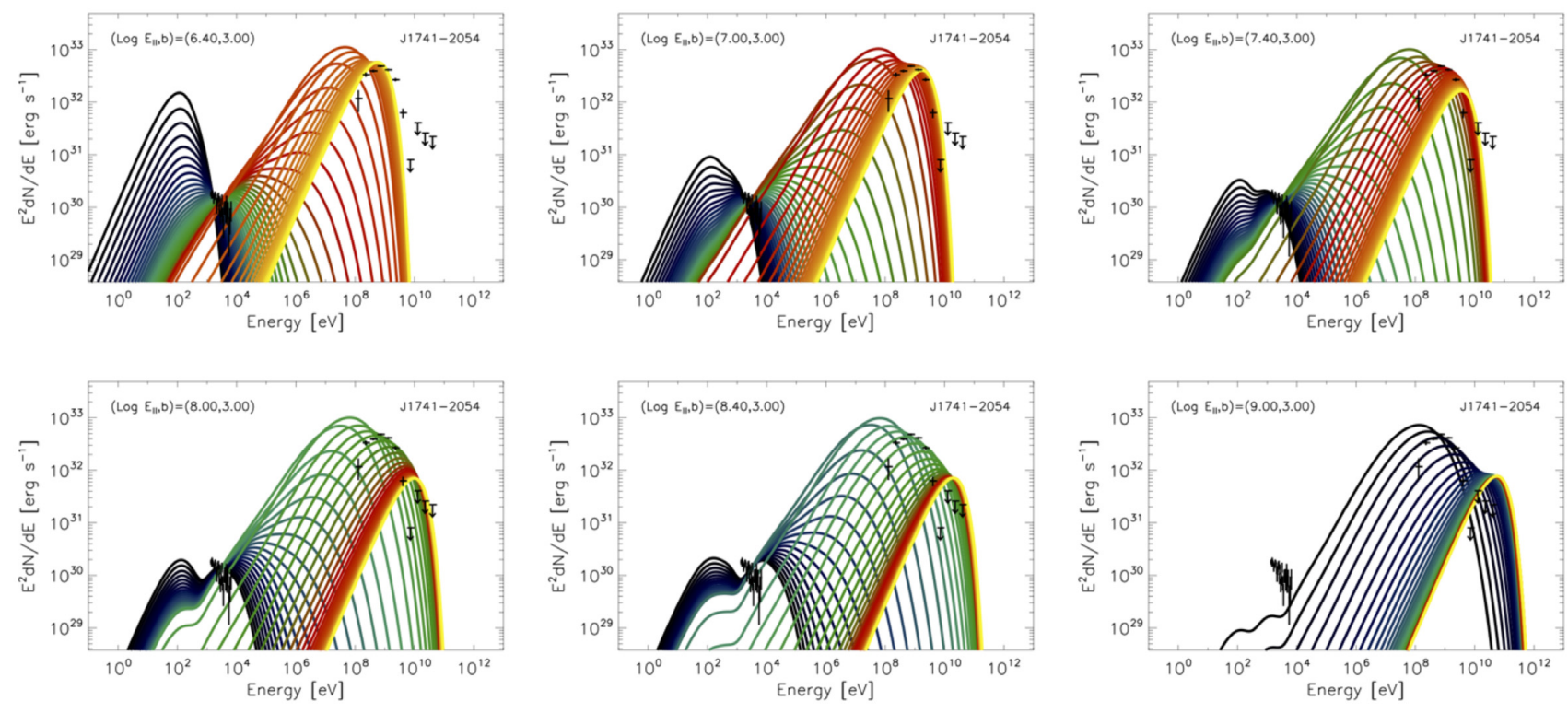

Figure 7. Example of model results for J1741-2054, searching for a common fit to whole spectrum from X-rays to gamma-rays. In these examples we vary $E_{\|}$(different in each panel, according to the legend) and $x_{0} / R_{\mathrm{lc}}$ (in the colour scale, see the text for details), for a fixed value of $b$ as given in the legend.
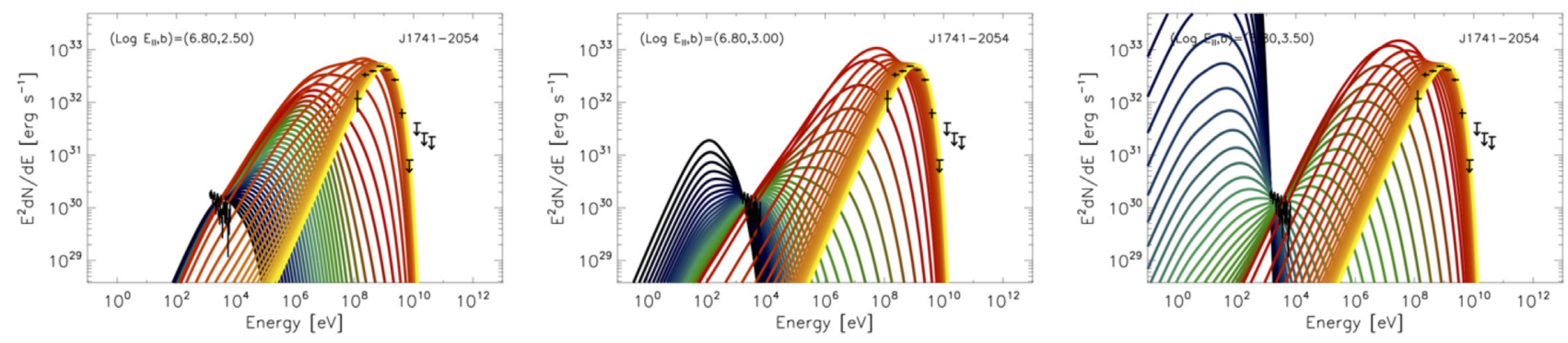

Figure 8. Same as Fig. 7, but showing different values of $b$ (different in each panel, according to the legend) instead of $E_{\|}$, which is fixed.

B1937+21, and J0218+4232, for which non-thermal X-ray emission has been discovered by Gotthelf \& Bogdanov (2017), show that a single set of parameters in our model can be enough to achieve a good fit. These MSPs are left out of the sample analysed in this work, since additional dedicated analysis of the gamma-ray data is needed to have a consistent data set. This will be presented elsewhere.

\section{SOLUTION AND INTERPRETATIONS OF THE FITTING MISMATCHES}

We shall first consider what kind of extension should the model be subject to in order to deal with the cases of Fig. 6. Two different interpretations for this extension are discussed next.

\subsection{The same $\left(E_{||}, b\right)$ but different $\left(x_{0} / R_{l c}, N_{0}\right)$ ?}

As commented above, the pair $\left(E_{\|}, b\right)$ represents physical parameters related to the region of acceleration, whereas the pair $\left(x_{0} / R_{\mathrm{lc}}\right.$, $N_{0}$ ) represents values related to the geometry: how uniform is the distribution of particles emitting radiation directed towards the observer, and how many particles does this distribution contain. As the gamma-ray data alone does not, in general, impose strong constraints on $b$, it can be used to define an $E_{\|}$range. That is, one can fix $b$ to a fiducial value (even the same for all pulsars) and get an approximate range and best value of $E_{\|}$that will be useful for fitting the gamma-ray data. Then, for that given value of $E_{\|}$one can find a best-fitting pair of $b$ and $x_{0} / R_{\mathrm{lc}}$ that better describes the X-ray data. The overall fitting can then be done in a loop, as conceptually represented in Fig. 9: with the best value of $E_{\|}$obtained from the gamma-ray fitting, we can span a range of $b$-values and fit the X-ray data, obtaining a best-fitting value of $b$, with the corresponding $x_{0} / R_{\mathrm{lc}}$ and $N_{0}$. With such value of $b$ one can then come back to the gamma-ray data set and fit it, to gather a new $E_{\|}$and the corresponding $x_{0} / R_{\mathrm{lc}}$ and $N_{0}$. The iterative process continues until convergence of both $E_{\|}$and $b$ values. For well-determined X-ray spectra, the loop can also be started from a fit to X-ray energies, leading to the same solution.

We note that in all of the cases of Section 2.3, as are shown in Fig. 6, with the same pair of values $\left(E_{\|}, b\right)$ and a different pair of values $\left(x_{0} / R_{\mathrm{lc}}\right.$ and $\left.N_{0}\right)$, we can fit both the X-ray and the gamma-ray data. These combined fittings are shown in Fig. 10. The value of the parameters in these (separate) fits are given in first panel of Table 2.

The strong(er) dependence of each part spectrum on a particular parameter ( $b$ for X-rays, and $E_{||}$for gamma-rays), especially visible when the model is confronted with data featuring a large energy coverage in these energy ranges and small error bars, does not imply at all that these separate fits are devoid of physical information. On the contrary, since here we fit the gamma-ray data in a completely independent way from the X-rays, there is nothing securing a-priori that this fit will not be above the observed X-ray flux level, being 


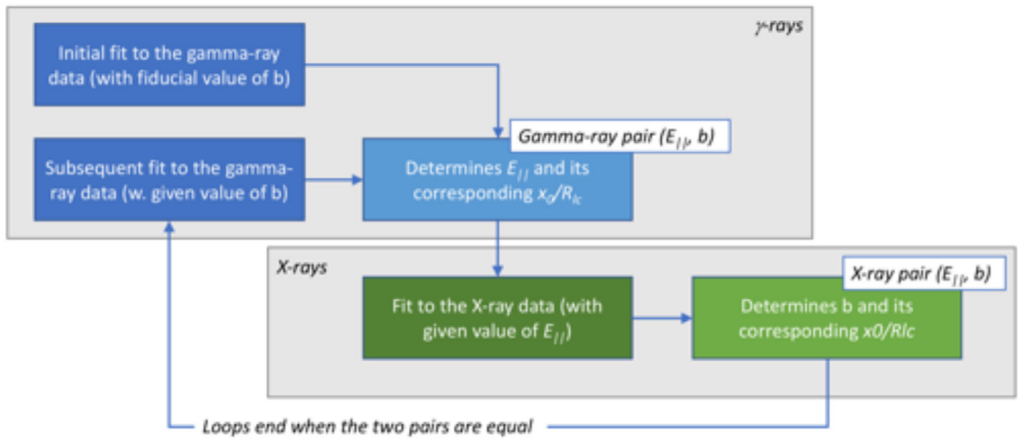

Figure 9. Conceptual representation of the fitting loop to determine the physical parameters of the accelerating region.
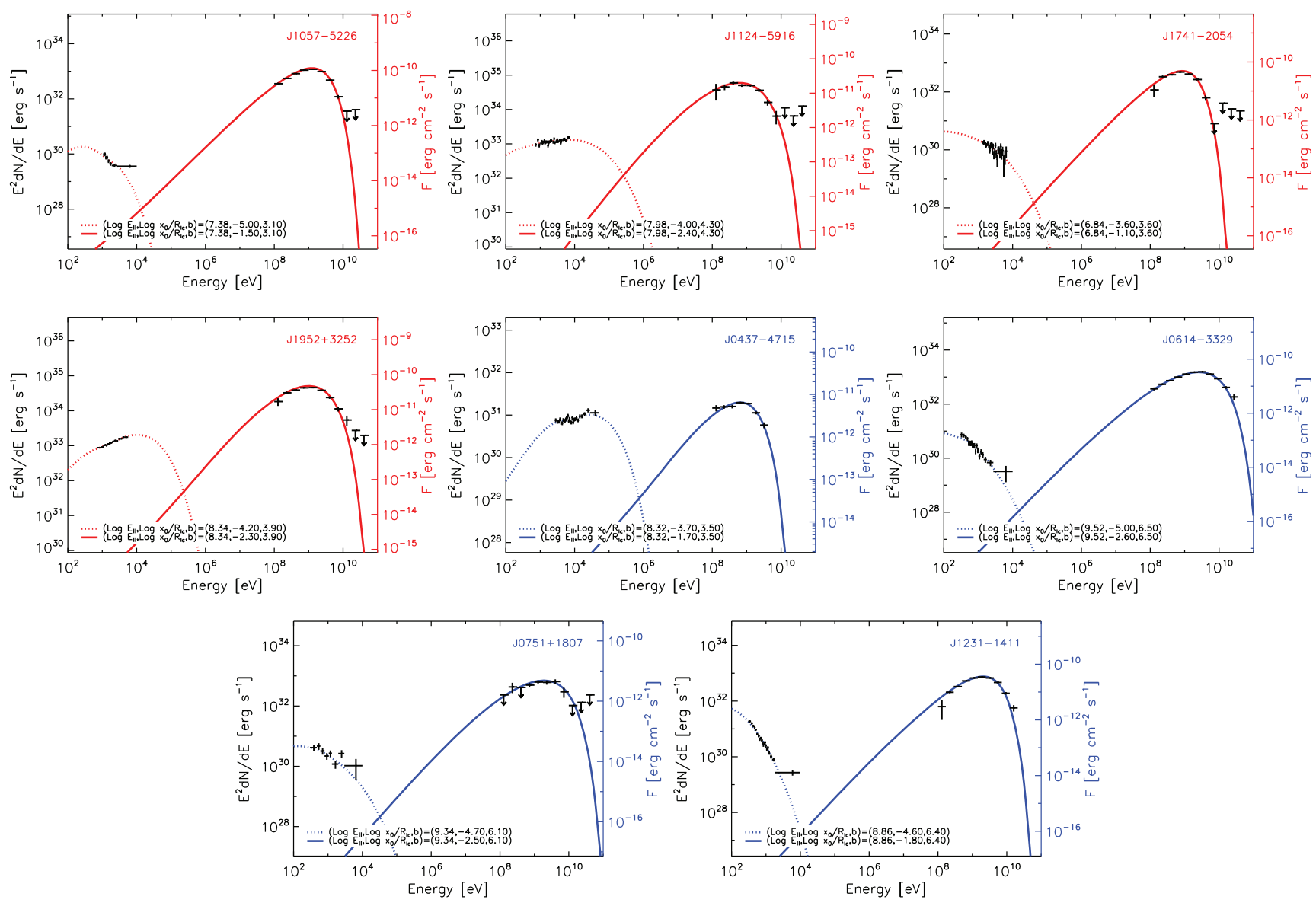

Figure 10. SEDs of PSRs (red) and MSP (blue) of Fig. 6 fitted when assuming two sets of parameters $\left(x_{0} / R_{\mathrm{lc}}, N_{0}\right)$, as could plausibly come from two regions of acceleration being visible, or from a more complex description of the particle distribution that emit radiation in direction to the observer. As noted in the legend the same value of $\left(E_{\|}, b\right)$ can be used to fit both parts of the SEDs. See the text for discussion.

incompatible. As an example, one can look at all the gamma-ray fits shown in Fig. 1 - dash-dotted lines, to see that, in most cases, the fit to the gamma-ray data already introduces a significant contribution in the X-ray band. This is not the case here: all fits to the gammaray data of Fig. 10 produce a very low yield in X-ray energies, underpredicting the observed X-ray SED and not interfering with the $\mathrm{X}$-ray fit obtained next. This is better shown in the spectral formation plots for these cases, which are exemplified in Fig. 11. It is shown there as to how the X-ray emission is again in all cases produced by synchrotron emission at the initial part of the particle's trajectories, and how the gamma-ray emission is in turn produced by the farther contributions. The extrapolation of each contribution into the other range (gamma-ray best-fit to $\mathrm{X}$-ray, and vice versa) is negligible.

It is also interesting to note that the $x_{0} / R_{\mathrm{lc}}$ value selected in the fit to the gamma-ray data is always larger than that selected in the fit to the X-ray data.

Note that if we used the model fitting only to the gamma-ray part of the SEDs of these pulsars (assuming that we do not yet know the X-ray part, and thus using a single set of parameters to describe the full SED) to infer which pulsars should be visible in X-rays, we would predict that the pulsars in Fig. 10 are not detectable. This implies that a selected sample of X-ray emitting pulsars starting 
Table 2. Results of piecewise spectral modelling ( $\mathrm{x}$ ( $\mathrm{g}$ ) refers to fitting only the X-ray (gamma-ray) data set). The panels correspond to Figs 10, 13, and 14 , respectively. In general, the gamma-ray fit is used to determine $E_{\|}$, whereas the X-ray fit is used to determine $b$. Exceptions occur (e.g. 0540$6919 \mathrm{~g}$, or $0357+3205 \mathrm{x}$ ) when such individual data sets are not currently constraining per se, and are discussed in the text.

\begin{tabular}{|c|c|c|c|c|c|c|}
\hline Type & Pulsar & $\begin{array}{c}D \\
(\mathrm{kpc})\end{array}$ & $\begin{array}{c}\log E_{\|} \\
\left(\mathrm{V} \mathrm{m}^{-1}\right)\end{array}$ & $b$ & $\log \left(x_{0} / R_{\mathrm{lc}}\right)$ & $\log N_{0}$ \\
\hline PSR & J1057-5226x & 0.30 & $7.38_{-\ldots}^{+\ldots}$ & $3.10_{-0.10}^{+2.40}$ & $-5.00_{-0.10}^{+0.90}$ & $31.74_{-0.78}^{+5.19}$ \\
\hline PSR & $\mathrm{J} 1057-5226 \mathrm{~g}$ & 0.30 & $7.38_{-0.06}^{+0.04}$ & $3.10_{-\ldots}^{+\ldots}$ & $-1.50_{-0.10}^{+0.10}$ & $30.43_{-0.03}^{+0.10}$ \\
\hline PSR & J1124-5916x & 5.00 & $7.98_{-\ldots}^{+\ldots}$ & $4.30_{-0.10}^{+0.10}$ & $-4.00_{-0.10}^{+0.10}$ & $37.47_{-0.41}^{+0.01}$ \\
\hline PSR & J1124-5916g & 5.00 & $7.98_{-0.46}^{+0.10}$ & $4.30_{-\ldots}^{+\ldots}$ & $-2.40_{-0.10}^{+0.60}$ & $33.02_{-0.37}^{+0.01}$ \\
\hline PSR & J1741-2054x & 0.30 & $6.84_{-\ldots}^{+\ldots}$ & $3.60_{-0.90}^{+1.90}$ & $-3.60_{-1.40}^{+0.10}$ & $36.37_{-5.83}^{+0.23}$ \\
\hline PSR & $\mathrm{J} 1741-2054 \mathrm{~g}$ & 0.30 & $6.84_{-0.10}^{+0.16}$ & $3.60_{-\ldots}^{+\ldots}$ & $-1.10_{-0.40}^{+0.30}$ & $30.33_{-0.16}^{+0.37}$ \\
\hline PSR & $\mathrm{J} 1952+3252 \mathrm{x}$ & 3.00 & $8.34_{-\ldots}^{+\ldots}$ & $3.90_{-0.10}^{+0.60}$ & $-4.20_{-0.10}^{+0.50}$ & $34.44_{-0.01}^{+1.99}$ \\
\hline PSR & $\mathrm{J} 1952+3252 \mathrm{~g}$ & 3.00 & $8.34_{-0.08}^{+0.02}$ & $3.90_{-\ldots}^{+\ldots}$ & $-2.30_{-0.10}^{+0.10}$ & $32.22_{-0.08}^{+0.01}$ \\
\hline MSP & J0437-4715x & 0.16 & $8.32_{-\ldots}^{+\ldots}$ & $3.50_{-0.10}^{+0.10}$ & $-3.70_{-0.10}^{+0.10}$ & $30.58_{-0.21}^{+0.01}$ \\
\hline MSP & J0437-4715g & 0.16 & $8.32_{-0.22}^{+0.22}$ & $3.50_{-\ldots}^{+\ldots}$ & $-1.70_{-0.30}^{+0.40}$ & $28.31_{-0.33}^{+0.18}$ \\
\hline MSP & J0614-3329x & 0.62 & $9.52_{-\ldots}^{+\ldots}$ & $6.50_{-0.10}^{+0.10}$ & $-5.00_{-0.10}^{+0.10}$ & $34.68_{-0.01}^{+0.01}$ \\
\hline MSP & $\mathrm{J} 0614-3329 \mathrm{~g}$ & 0.62 & $9.52_{-0.08}^{+0.02}$ & $6.50_{-\ldots}^{+\ldots}$ & $-2.60_{-0.10}^{+0.10}$ & $29.39_{-0.04}^{+0.01}$ \\
\hline MSP & $\mathrm{J} 0751+1807 \mathrm{x}$ & 1.10 & $9.34_{-\ldots}^{+\ldots}$ & $6.10_{-0.80}^{+0.40}$ & $-4.70_{-0.30}^{+0.10}$ & $34.49_{-1.59}^{+0.90}$ \\
\hline MSP & $\mathrm{J} 0751+1807 \mathrm{~g}$ & 1.10 & $9.34_{-0.58}^{+2.16}$ & $6.10_{-\ldots}^{+\ldots}$ & $-2.50_{-2.30}^{+1.00}$ & $29.28_{-0.74}^{+0.46}$ \\
\hline MSP & J1231-1411x & 0.42 & $8.86_{-\ldots}^{+\ldots}$ & $6.40_{-0.10}^{+0.10}$ & $-4.60_{-0.10}^{+0.10}$ & $36.08_{-0.31}^{+0.27}$ \\
\hline MSP & $\mathrm{J} 1231-1411 \mathrm{~g}$ & 0.42 & $8.86_{-0.04}^{+0.06}$ & $6.40_{-\ldots}^{+\ldots}$ & $-1.80_{-0.10}^{+0.10}$ & $28.82_{-0.10}^{+0.05}$ \\
\hline PSR & $\mathrm{J} 0357+3205 \mathrm{x}$ & 0.83 & $6.86_{-\ldots}^{+\ldots}$ & $3.00_{-\ldots}^{+\ldots}$ & $-3.30_{-0.10}^{+0.10}$ & $32.48_{-0.01}^{+0.01}$ \\
\hline PSR & $\mathrm{J} 0357+3205 \mathrm{~g}$ & 0.83 & $6.86_{-0.14}^{+0.12}$ & $3.00_{-\ldots}^{+\ldots}$ & $-1.30_{-0.20}^{+0.40}$ & $31.25_{-0.31}^{+0.10}$ \\
\hline PSR & J1513-5908x & 4.40 & $7.00_{-\ldots}^{+\ldots}$ & $3.10_{-0.10}^{+0.10}$ & $-3.40_{-0.10}^{+0.10}$ & $33.91_{-0.01}^{+0.01}$ \\
\hline PSR & $\mathrm{J} 1513-5908 \mathrm{~g}$ & 4.40 & $7.00_{-0.02}^{+0.10}$ & $3.10_{-\ldots}^{+\ldots}$ & $-1.90_{-0.10}^{+0.10}$ & $34.34_{-0.08}^{+0.01}$ \\
\hline PSR & J1709-4429x & 2.60 & $8.54_{-\ldots}^{+\ldots}$ & $3.50_{-0.50}^{+0.60}$ & $-5.00_{-0.10}^{+1.70}$ & $33.44_{-1.50}^{+1.91}$ \\
\hline PSR & J1709-4429g & 2.60 & $8.54_{-0.02}^{+0.02}$ & $3.50_{-\ldots}^{+\ldots}$ & $-2.80_{-0.10}^{+0.10}$ & $33.34_{-0.01}^{+0.01}$ \\
\hline PSR & J1826-1256x & 1.55 & $8.08_{-\ldots}^{+\ldots}$ & $3.80_{-\ldots}^{+\ldots}$ & $-3.20_{-0.10}^{+0.10}$ & $34.24_{-0.01}^{+0.01}$ \\
\hline PSR & $\mathrm{J} 1826-1256 \mathrm{~g}$ & 1.55 & $8.08_{-0.16}^{+0.18}$ & $3.80_{-\ldots}^{+\ldots}$ & $-2.40_{-0.20}^{+0.20}$ & $32.60_{-0.12}^{+0.05}$ \\
\hline PSR & J1833-1034x & 4.10 & $8.04_{-\ldots}^{+\ldots}$ & $3.40_{-0.10}^{+0.10}$ & $-3.70_{-0.10}^{+2.40}$ & $33.32_{-0.01}^{+2.57}$ \\
\hline PSR & J1833-1034g & 4.10 & $8.04_{-0.48}^{+2.14}$ & $3.40_{-\ldots}^{+\ldots}$ & $-2.20_{-2.20}^{+0.70}$ & $32.55_{-0.43}^{+0.32}$ \\
\hline PSR & $\mathrm{J} 1836+5925 \mathrm{x}$ & 0.30 & $7.76_{-\ldots}^{+\ldots}$ & $3.30_{-0.20}^{+1.70}$ & $-3.90_{-0.59}^{+0.10}$ & $32.01_{-0.93}^{+2.70}$ \\
\hline PSR & $\mathrm{J} 1836+5925 \mathrm{~g}$ & 0.30 & $7.76_{-0.02}^{+0.02}$ & $3.30_{-\ldots}^{+\ldots}$ & $-2.00_{-0.10}^{+0.10}$ & $30.88_{-0.01}^{+0.01}$ \\
\hline PSR & $\mathrm{J} 2030+4415 \mathrm{x}$ & 0.88 & $7.46_{-\ldots}^{+\ldots}$ & $3.70_{-0.10}^{+0.10}$ & $-3.80_{-0.10}^{+0.10}$ & $34.78_{-0.01}^{+0.01}$ \\
\hline PSR & $\mathrm{J} 2030+4415 \mathrm{~g}$ & 0.88 & $7.46_{-0.02}^{+\ldots .}$ & $3.70_{-\ldots}^{+\ldots}$ & $-2.00_{-0.10}^{+0.10}$ & $31.65_{-0.01}^{+0.01}$ \\
\hline PSR & $\mathrm{J} 2055+2539 \mathrm{x}$ & 0.62 & $7.10_{-\ldots}^{+\ldots}$ & $2.80_{-\ldots}^{+\ldots}$ & $-4.70_{-0.10}^{+0.30}$ & $30.84_{-0.63}^{+2.33}$ \\
\hline PSR & $\mathrm{J} 2055+2539 \mathrm{~g}$ & 0.62 & $7.10_{-0.18}^{+0.14}$ & $2.80_{-\ldots}^{+\ldots}$ & $-1.30_{-0.30}^{+0.60}$ & $30.46_{-0.32}^{+0.22}$ \\
\hline PSR & $\mathrm{J} 0534+2200 \mathrm{x}$ & 2.00 & $8.94_{-\ldots}^{+\ldots}$ & $5.10_{-0.10}^{+0.10}$ & $-4.30_{-0.10}^{+0.10}$ & $38.89_{-0.01}^{+0.01}$ \\
\hline PSR & $\mathrm{J} 0534+2200 \mathrm{~g}$ & 2.00 & $8.94_{-0.02}^{+0.02}$ & $5.10_{-\ldots}^{+\ldots}$ & $-3.00_{-0.10}^{+0.10}$ & $33.29_{-0.01}^{+0.01}$ \\
\hline PSR & J0540-6919x & 49.70 & $8.00_{-0.02}^{+0.02}$ & $5.40_{-0.10}^{+0.10}$ & $-3.70_{-0.10}^{+0.10}$ & $40.48_{-0.01}^{+0.01}$ \\
\hline PSR & J0540-6919g & 49.70 & $8.00_{-\ldots}^{+\ldots . \ldots}$ & $5.40_{-\ldots}^{+\ldots}$ & $-2.10_{-\ldots}^{+\ldots}$ & $34.08_{-\ldots}^{+\ldots}$ \\
\hline
\end{tabular}

from a gamma-ray model fitting maybe incomplete, but we shall not waste X-ray satellites observation time searching for gammaray selected pulsars: those pulsars that may ultimately not be fitted well by the gamma-ray derived parameters, despite being noted by the gamma-ray fitting as detectable in X-rays, can be more (not less) luminous.

\subsection{Two regions of acceleration visible?}

One obvious possibility for interpreting how two populations of particles subject to the same $\left(E_{\|}, b\right)$ but featuring a different $\left(x_{0} / R_{\mathrm{lc}}\right.$, $N_{0}$ ) can intervene in the generation of the SED is to entertain that there are two acceleration regions visible to the observer, and that they sustain a different geometrical orientation.

Indeed, if there are two regions of acceleration, it is reasonable to suppose that they shall have equal physical properties (i.e. the same pair of intrinsic parameters, $\left.\left(E_{\|}, b\right)\right)$, corresponding to the same pulsar, and located in symmetrical positions in the pulsar magnetosphere. However, if both are visible, they will likely present a different pair of $\left(x_{0} / R_{\mathrm{lc}}, N_{0}\right)$ for describing the relevant population of particles emitting radiation towards the observer, given that the latter is in a different direction with respect to each accelerating region.

Some observers would see the emission mostly coming from one acceleration region, the geometry of which would be more favourable: during a larger part of the trajectories there would be particles moving (and emitting) in the direction of the observer. This would lead to a (relatively) more uniform distribution, and thus to a larger $x_{0} / R_{\mathrm{lc}}$. The geometry of the other accelerating region may be less favourable, such that the distribution of particles emitting towards the observer is more skewed, leading to a smaller $x_{0} / R_{\mathrm{lc}}$ The fact that the $x_{0} / R_{\mathrm{lc}}$ value selected in the fit to the gamma-ray data is larger than that selected in the fit to the X-ray data is consistent with this geometrical interpretation: the gamma-ray emission is produced further along the trajectories of particles, thus from the more uniform population, and we see particles reaching to a higher energies. Because of that, the $x_{0} / R_{\mathrm{lc}}$ value fitting the gamma-ray observations is larger (the contrast is smaller) than the one chosen by the X-ray data fit.

This concept is represented in the left-hand panel of Fig. 12. In that figure, the location of several possible acceleration regions are noted with different colours, assuming that two symmetric locations appear. Several different observers (colour coded) are noted as an example with respect to which assess the geometrical configuration. For instance, in Fig. 12, the dark and light red represent pairs of regions of acceleration whose geometrical orientation may be leading to different values of $x_{0} / R_{\mathrm{lc}}$ and $N_{0}$, as noted in the legend. The cones are representing the direction of emission at different parts of the trajectories of particles: they are wider at the initial part where synchrotron domination occurs via the loss of perpendicular angular momentum, and smaller in regions where the trajectory is more curvature-dominated. Note that even when existing, geometry would not always allow seeing the two regions of acceleration (see the violet case in Fig. 12, where the represented geometry would lead to only one region of acceleration being visible). Finally, in cases in which both accelerating regions are similarly oriented (e.g. the green case of the conceptual left-hand panel of Fig. 12), or in cases in which only one region is visible, a single set of $\left(E_{\|}, b\right.$, $x_{0} / R_{\mathrm{lc}}, N_{0}$ ) parameters would suffice to represent the SED. This interpretation seems a proper explanation for all cases, but has additional consequences on the expected light curves that we put in context below, after discussing an alternative.

\subsection{One region of acceleration with a more complex particle distribution?}

There is a second possible interpretation for a double set of $\left(x_{0}\right.$, $N_{0}$ ) parameters. The distribution of the particles emitting towards us, $\left(\mathrm{d} N_{e} / \mathrm{d} x\right)$, may be different at different energies, even when only one region of emission is visible, and therefore may not be well-describable by a single set of parameters $\left(x_{0}, N_{0}\right)$ as used in equation (2).

For the relativistic particles we are considering $\left(\Gamma>10^{3}\right)$, the opening of the emission cone centred around the direction of motion 

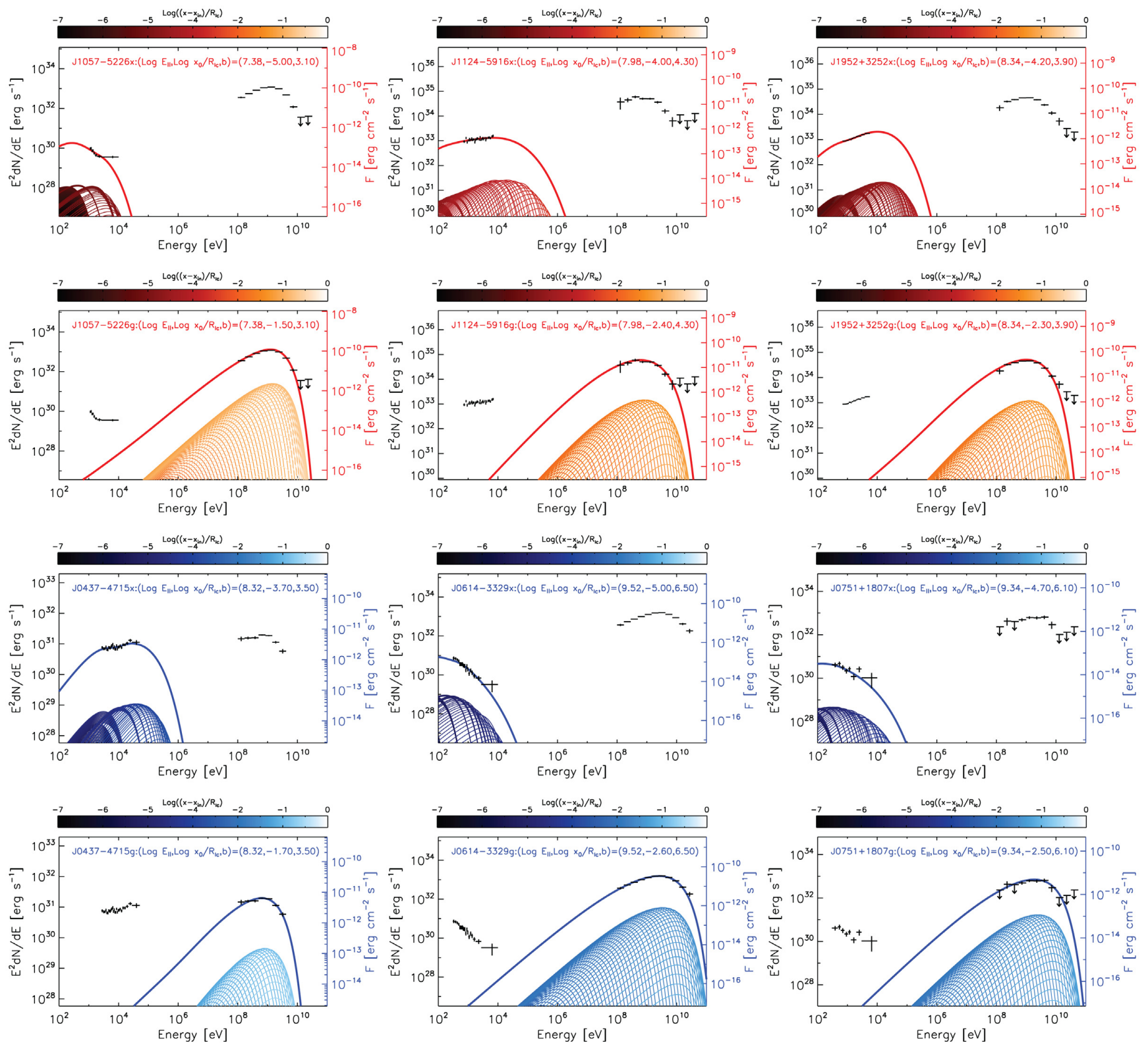

Figure 11. Examples for the formation of the SEDs in cases where two sets of $\left(x_{0} / R_{\mathrm{lc}}, N_{0}\right)$ are needed to represent the full SED. Two panels are shown for each pulsar, associated to the X-ray and gamma-ray fits.

(at which boundaries the radiation emitted peaks and along which the angular distribution of the radiation is spread), despite being energy-dependent, is vanishingly small at all energies. This implies that photons are always emitted in the instantaneous direction of motion of the particles. When the pitch angle tends to zero, which is valid in all cases where curvature dominates synchrocurvature emission (Viganò et al. 2015a), all radiation points in the tangent direction. But this is not the case if synchrotron emission dominates synchrocurvature, when large pitch angles are found. The gyroaveraged emission distribution, for a given position along the line, describes a circle centred around the tangent direction, with a radius given by the particle pitch angle. Thus, particles that are not moving in the direction to the observer, may still radiate photons that do when the pitch angle is large. To correctly describe that such a relatively large number of particles are able to emit in the direction to the observer in the synchrotron-dominated synchrocurvature regime, a large contrast is needed in equation (2). However, this contrast may incorrectly underestimate the number of particles emitting towards the observer at larger energies. This is conceptually represented in the right-hand panel of Fig. 12. Given that the sum of two populations, $p_{1}$ and $p_{2}$, each with parameters $\left(x_{0}^{p_{1}} / R_{l c}, N_{0}^{p_{1}}\right)$ and $\left(x_{0}^{p_{2}} / R_{l c}, N_{0}^{p_{2}}\right)$ cannot be encompassed in a single set of new parameters $\left(x_{0}, N_{0}\right)$, the use of single distribution may be a bad approximation to the relevant number of particles.

We note that in this scheme, the use of two sets of values $\left(x_{0}, N_{0}\right)$ to describe a high (and more uniform) and low (and more skewed) intervening particle population is also an approximation, of course. It is better than using just one set, but still an approximation to a more complex situation where ultimately $\left(\mathrm{d} N_{e} / \mathrm{d} x\right)$ is a function of 

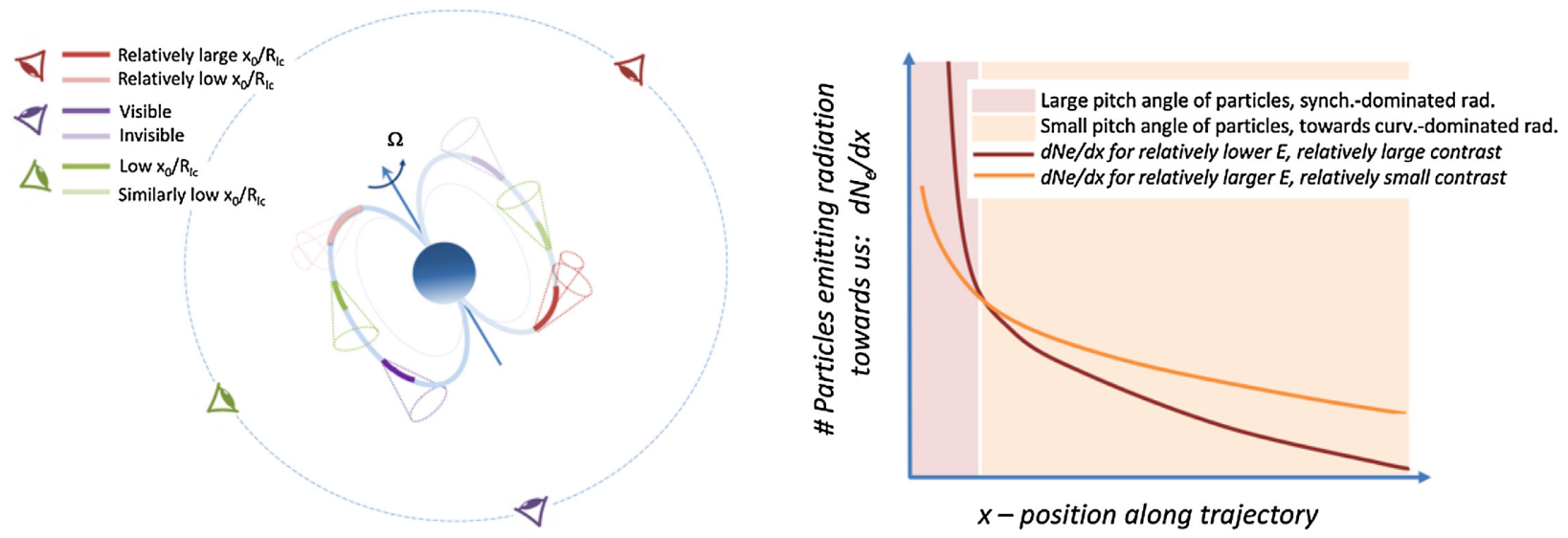

Figure 12. Left-hand panel: Conceptual representation of the effect of the orientation to the observer with regards to the parameter $x_{0} / R_{\mathrm{lc}}$. We use (for the sake of keeping the plot simple) locations of the acceleration regions along a closed field line to convey the idea only. The positions of the two accelerating regions, and the corresponding observer, are colour-coded. Right-hand panel: Conceptual representation of the possible inability of a single set of $x_{0} / R_{\mathrm{lc}}, N_{0}$ parameters to describe the population of particles emitting towards the observer at all energies. The total number of particles would be the sum of the two curves. See the text for further discussion.

energy; there is only one particle population and one accelerating region in this interpretation. We come back to this concept when discussing Crab below.

\subsection{Relation of these interpretations with the pulsar light curves}

Light curves cannot be directly modelled by our SED approach without further significant extensions. Several effects related to the emission process itself and to where this emission happens along the particles trajectories concur to shape the light curves beyond the location of the acceleration regions. However, we can already note that the two interpretations suggested above should be qualitatively reflected in different light-curve properties.

For instance, the X-ray and gamma-ray emission, if coming predominantly from different regions of acceleration as in the lefthand panel of Fig. 12, should appear phase-shifted. For at least some cases (but not all) where simultaneous light curves have been measured, this indeed appears to occur: such are for instance the cases of J1057-5226 or J1741-2054, where the gamma-ray and Xray peaks are phase-offset by roughly a half rotation, e.g. see fig. 3 of Marelli et al. (2014).

Instead, if both the X-ray and the gamma-ray emission come from the same emission region, as would be the case in the righthand panel of Fig. 12 where a more complex particle distribution is needed to describe the population emitting towards us, there should be a smaller phase shift between the low- and high-energy light curve.

Whereas we are not yet offering a first-principle computation of the light curves predicted by our model, we note that as commented by Marelli et al. (2014), no model has been able to account for offsets between gamma-ray and X-ray peaks before. It seems that this fact can be qualitatively explained here: we offer a plausible interpretation by which not always the X-ray and gamma-ray emission come from the same place.

We also note that even in the case of pulsars that can be fitted by a single set of parameters, if particles are all injected at the same location there should be a delay between the emission of X-rays and that of gamma-rays (see the right set of panels of Fig. 3 for an explicit computation). This delay could be a significant fraction of the period of the pulsar, what would ultimately introduce a phase shift.

\section{ANOTHER LOOK TO THE BETTER-FITTED SEDS}

\subsection{A double fitting approach for the arguably good SED fittings}

Using the fitting approach described above for all the pulsars signalled in Fig. 5, we also note that the model is able to fit better both the X-ray and gamma-ray data. The addition of only these two extra parameters in the model (the extra set of $x_{0}, N_{0}$ ) indeed promotes qualitative improvements in all these cases.

The double fits are shown in Fig. 13, and the parameters for these fits are stated in the second panel of Table 2. A few comments may be in order to clarify the values there. Especially due to the energy coverage, shape, and error bars of the current X-ray data set, in some of the cases, e.g. PSR J0357+3205, J1826-1256, or $\mathrm{J} 2055+2539$, neither the X-ray nor the gamma-ray data sets by themselves promote a significant constraint on $b$, i.e. essentially all $b$-values explored are admissible within $1 \sigma$. This would of course change in case additional data are available. In such cases, then we fixed a fiducial value: $b=3.00$ for $\mathrm{J} 0357+3205, b=$ 3.80 for $\mathrm{J} 1826-1256$, and $b=2.80$ for $\mathrm{J} 2055+2539$ (that are selected as the best solutions) respectively, to show that indeed the same possible solution of a double fitting can occur. This is why there are no errors in the $b$ values of the $\mathrm{X}$-ray fittings in Table 2 for these cases. A hard X-ray observation in these (and essentially in all) cases would be extremely relevant to fix the model and the physics it contains better. These cases are similar to that of J1741-2054 treated before (see Table 2), where the admissible range of $b$ was also large, and we assumed the best-fitting solution to gather the $b$-value used in the gamma-ray fitting. Finally, in the case of PSR J2030+4415, the gamma-ray fitting only provides a lower limit on $E_{\|}$which is what we use to represent the common solution used in both $\mathrm{X}$ and gamma-ray data.

The spectral formation in both components is similar in all of these cases to that exemplified in Fig. 11. 

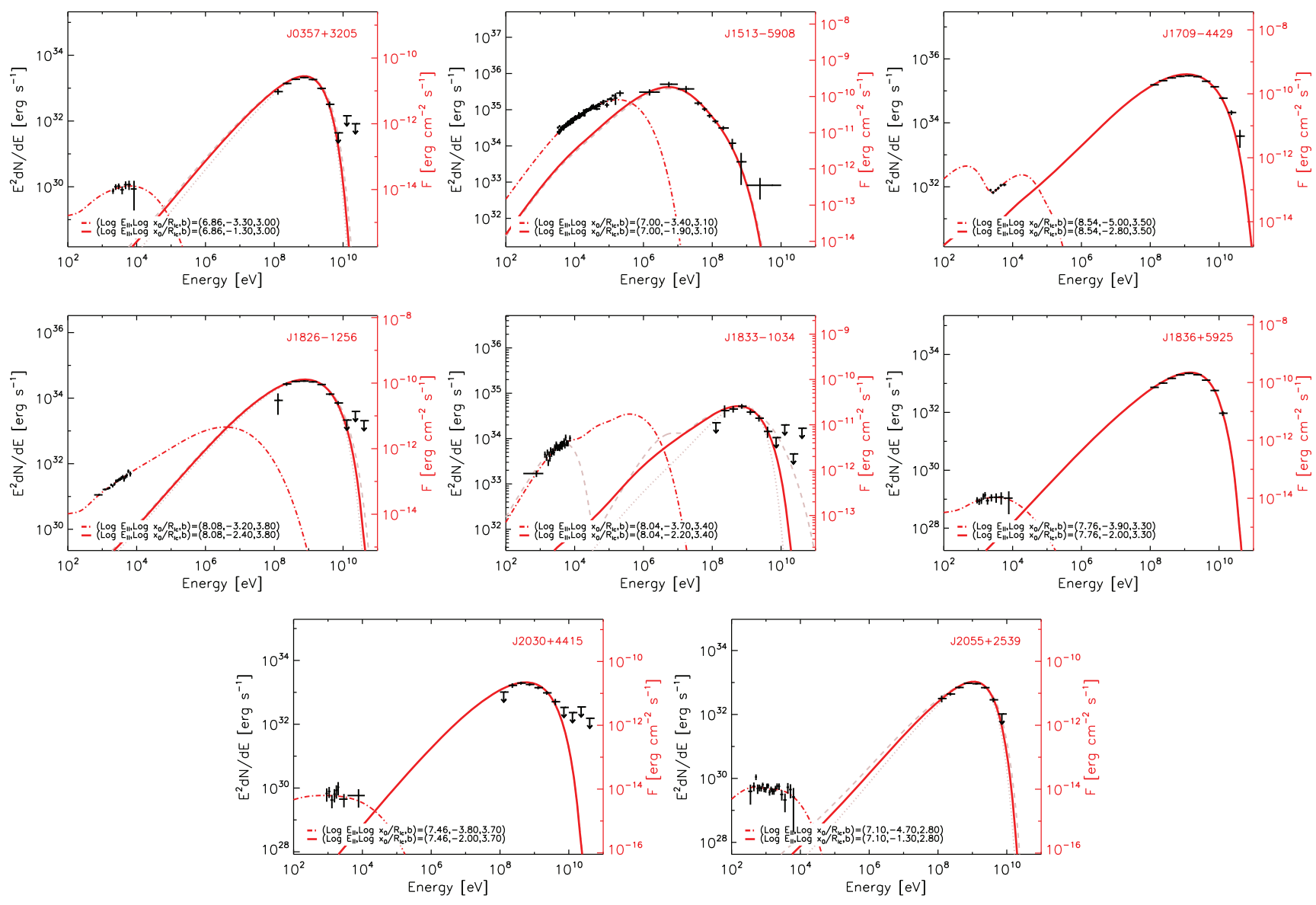

Figure 13. SEDs of the pulsars of Fig. 5 analysed under the perspective of a separate fitting to the X-ray and gamma-ray part of the spectrum. Also here, and as noted in the legend, the same value of the pair $\left(E_{\|}, b\right)$ can be used to fit both parts of the SEDs. See the text for discussion.

\subsection{Is there degeneracy in the fitting of well-determined SEDs?}

In principle, if for a given SED, a single set of parameters $\left(x_{0}\right.$, $N_{0}$ ) does fit qualitatively well, this is better (less parameters, more elegant solution) than having to use two sets. But can one have e.g. two accelerating regions, with different $x_{0} / R_{\mathrm{lc}}$ values and still have good (and different) spectral fits in the cases treated in Fig. 1?

We studied these issues with the best measured cases, among them PSR J0007+7303, Vela (J0835-4510), Geminga (0633+1746), and J1813-1246. We found that in all of these cases, the iterative loop for fitting used above for the less adequate single-set cases (see Fig. 9) leads to a solution that is compatible within $1 \sigma$ to the common X-ray and gamma-ray fit of Fig. 1. For instance, in the case of $\mathrm{J} 0007+7303$, the X-ray fit using the $E_{||}$value obtained from a gamma-ray fit would put no constraint on $b$, providing equally good solutions in the range explored. For one of these values of $b$, then, we would reproduce the common X-ray / gamma-ray fit. In the case of Geminga, fixing the $E_{\|}$value as obtained from the gammaray only fit, the best solution for the X-ray data already chooses $b=$ 2.5 , and thus the iteration would exactly reproduce the common X-ray/gamma-ray fit. Vela and J1813-1246 are cases for which one can argue a priori that a separate X-ray and gamma-ray fit may improve the global appearance. For instance, for Vela the single set model correctly reproduces the general trend and the flatness of a well-determined low-energy X-ray spectrum, but misses the lowest energy X-ray data point and seems to underpredict the rising trend in the hard X-ray domain. However, a separate fit to the two components does no better, with the loop also converging to the same (single) solution. For J1813-1246, one can also argue that the model is missing the lowest energy gamma-ray point (but see the comment above regarding the caution needed to reject a model based on this point only). In addition, for J1813-1246 the difference between the gamma-ray only fit (using the fixed value of $b=2.85$ ) and the common X-ray/ gamma-ray fit is relatively large (providing electric fields from $E_{\|}=8.20$ to 9.22 ), which may promote hopes that a separate fitting would find a better alternative solution. We tried the separate fitting starting from both values of $E_{\|}$. However, the iterative loop would either converge to a solution where the common X-ray/ gamma-ray fit would be admissible within the uncertainty (for the larger value of $E_{\|}$) or do not find a good fit for both parts of the spectrum at once. This same situation also happens in other cases with a lesser quality of observational data, like J1357-6429. For J1813-1246, we have also tried to fit the gamma-ray data only ranging on the $b$ value, to find that the best parameter is already close (or the same within errors) to that in the common X-ray and gamma-ray fit, thus providing a very good representation of the X-ray data already (although this fit is not optimized since the X-ray data are not taken into account and thus the fit is not preferred in comparison to the common one).

These detailed fitting studies led us to conclude that there are no obvious separate fitting solutions to the best-determined cases in Fig. 1 that qualitatively improve those shown there, minimizing the possible degeneracy issues in these cases. 


\section{CRAB AND THE CRAB TWIN}

The Crab and the Crab twin present a relatively flat SED across several orders of magnitude in energy in the X-ray regime, at a level comparable to the gamma-ray yield. This is a distinctive feature. As fig. 2 of Coti Zelati et al. (2019) shows, most other pulsars present SEDs that are either clearly rising or clearly decreasing with energy. Those few cases for which the X-ray part of the SED is flatter, present a much lower yield there in comparison with gamma-rays, except perhaps for a few MSPs/ For Crab, the X-ray yield actually exceeds that in gamma-rays.

In our model, we find that it is impossible that a single set of parameters $\left(E_{\|}, b, x_{0}, N_{0}\right)$ produce a qualitatively good fit across the full energy range for Crab or the Crab Twin, similar to the other cases of Fig. 6.

Given the quality of the data set we profit Crab as a testbed to see whether variations in the parameters appearing in equation (1) but kept fixed in our modelling would be of any help in providing a common fit. In short, the answer is no, as expected. Details about some of these tests are given next.

\subsection{Crab}

The data we use here, apart from the gamma-ray measurements from Abdo et al. (2013), comes from the compilation of Bühler \& Blandford (2014), the $\mathrm{MeV}$ data from Kuiper et al. (2001), and the infrared (IR) to ultraviolet (UV) measurements reported in Sollerman et al. (2000) and Tziamtzis, Lundqvist \& Djupvik (2009). For the TeV data see Aliu et al. (2008, 2011), Aleksić et al. (2011), and Ansoldi et al. (2016). This is the most complete sample for the phase averaged spectrum of a pulsar, and fitting them in a model has proven difficult.

The best-fitting SED coming from our model is shown in Fig. 14. The values of the best-fitting parameters are given in the last panel of Table 2. We fitted the Fermi gamma-ray data at high energies (and got a qualitatively good fit also for the lower gamma-ray data set as measured by EGRET) and the optical and keV RXTE data at lower energies. Especially the lower energy fit is qualitatively good, although we find that the flatness of the spectrum in the X-ray regime requires a relatively large value of $b$, close to those found above for MSPs. In comparison, an individual (not iterative) fitting of the gamma-ray spectrum would rather prefer a lower value of $b$, although such values cannot deal at all with the X-ray data. Instead, a larger value of $b$ can provide a good match to the X-ray / optical data set, and still be reasonably close to the high energy portion of the spectrum.

The best solution found under the same scheme used for the rest of the pulsars in this paper is shown in the left-hand panels of Fig. 14. There, we show the best-fitting together with the influence of the contrast for each portion of the solution (the first three panels). The solution found in the first (top, left) panel comes from the best-fitting components of the green plots representing the contrast influence. Note that difference between the curves plotted in the second and third panels is just in the normalization, the corresponding (colourcoded) curves have the same $\left(E_{\|}, b\right)$ and also $x_{0} / R_{\mathrm{lc}}$ values. The normalization, instead, is automatically chosen for each of these curves in Fig. 14 in order to provide the best fit - for that particular set of parameters - to either the X-ray (second from top) or the gamma-ray (third from top) emission. Also here, the latter plots are useful to understand why a single solution cannot fit both sets of data (this is similar to the previously studied cases). Finally, the last two left-hand column plots show the spectral contributions to the spectrum of the low (mostly synchrotron) and high-energy (mostly synchrocurvature to curvature) radiation.

We note that the $\mathrm{MeV}$ data is underpredicted in this model (summing the two components there is a underprediction in the $\mathrm{MeV}$ range). As the Crab light curve is correlated at different energy regimes, the interpretation described in the right-hand panel of Fig. 12 would justify the use of two sets of $\left(x_{0}, N_{0}\right)$ as an approximation to the real population of particles emitting radiation towards us. Then, we pose that the failure to reproduce well the MeV data may just indicate that this representation is still not enough for a good accounting of the particle population. Obviously, the transition from one set of geometrically related parameters to the other should be a continuous process. This may become more visible for nearby pulsar with a large rotational power, which generate more particles flowing in the magnetosphere, becoming increasingly more difficult to represent the particle distribution. It might be natural to think that to represent the particle population the approximation of two sets is also not enough. The quality of the data set for Crab would surface this. A better representation of the particle distribution (still with the same $\left.\left(E_{\|}, b\right)\right)$ may thus overcome this caveat. In fact, just to emphasize this possibility we notice that using the same $\left(E_{\|}, b\right)$ as given in Table 2 to search for a fit to the $\mathrm{MeV}$ data with (i.e. with all parameters fixed except for $x_{0}$-and $N_{0}-$ ) we find a match for a value of $\left(\log \left(x_{0} / R_{\mathrm{lc}}\right), \log N_{0}\right)=(-3.80,36.65)$, intermediate between that needed for the low- and high-energy parts of the SED. This contribution would naturally arise in a continuous representation of the distribution of particles emitting towards the observer, and the fit would not interfere with the prediction at hard X-rays, or $\mathrm{X}$-rays, nor with that at higher gamma-ray energies, which would continue being produced as described. The total yield of such a model is represented in Fig. 15. Formally, it contains seven free parameters, the two intrinsically related pulsar properties $\left(E_{\|}, b\right)$, and three sets of $\left(x_{0}, N_{0}\right)$ used to represent the particle population one of the $N_{0}$ can be considered fixed, since one can fit one of the SED portions only in its shape and normalize the rest referencing to it. The parameters used to represent an energy-dependent $\mathrm{d} N / \mathrm{d} x$ in a piecewise energy-independent manner can probably be reduced if using a better representation of the particle distribution. This remains to be studied, and represents a challenge. The increased number of parameters is to be put in context to those needed in other models: for instance, Lyutikov (2013) uses nine parameters to determine the particle population spectrum and its location to generate the Crab spectrum via a cyclotron self-Compton model. The latter model correctly describes the Crab's TeV emission (see next) but underpredicts the optical and IR yield.

We note that our Crab model underpredicts the TeV data. Could this be modelled similar to the $\mathrm{MeV}$ part of the spectrum (i.e. using the same $\left(E_{\|}, b\right)$ pair) just discussed? We find it is indeed possible to represent the $\mathrm{TeV}$ component with a small population of particles emitting towards us, but requiring a larger accelerating field, $\log E_{\|} \gtrsim 9.84$. In this scheme, the $\mathrm{TeV}$ emission would be curvature, and it thus does not rule out that curvature can still be behind the largest photon energies if an enhancement of $E_{\|}$is possible. However, such value of $E_{\|}$would in turn be inadequate for representing the X-ray to gamma-ray spectrum. The need of a different $E_{\|}$cannot be encompassed in the interpretation posed above, where just two parameters define its intrinsic physics. We tested whether this conclusion would be affected by a different assumption on $x_{\text {in }}$ or $x_{\text {out }}$, and the size of the accelerating region, finding that this is not the case. Thus, these data could be produced somewhere else still via curvature with an enhanced $E_{\|}$, or by a different process altogether, e.g. see Aharonian, Bogovalov \& 

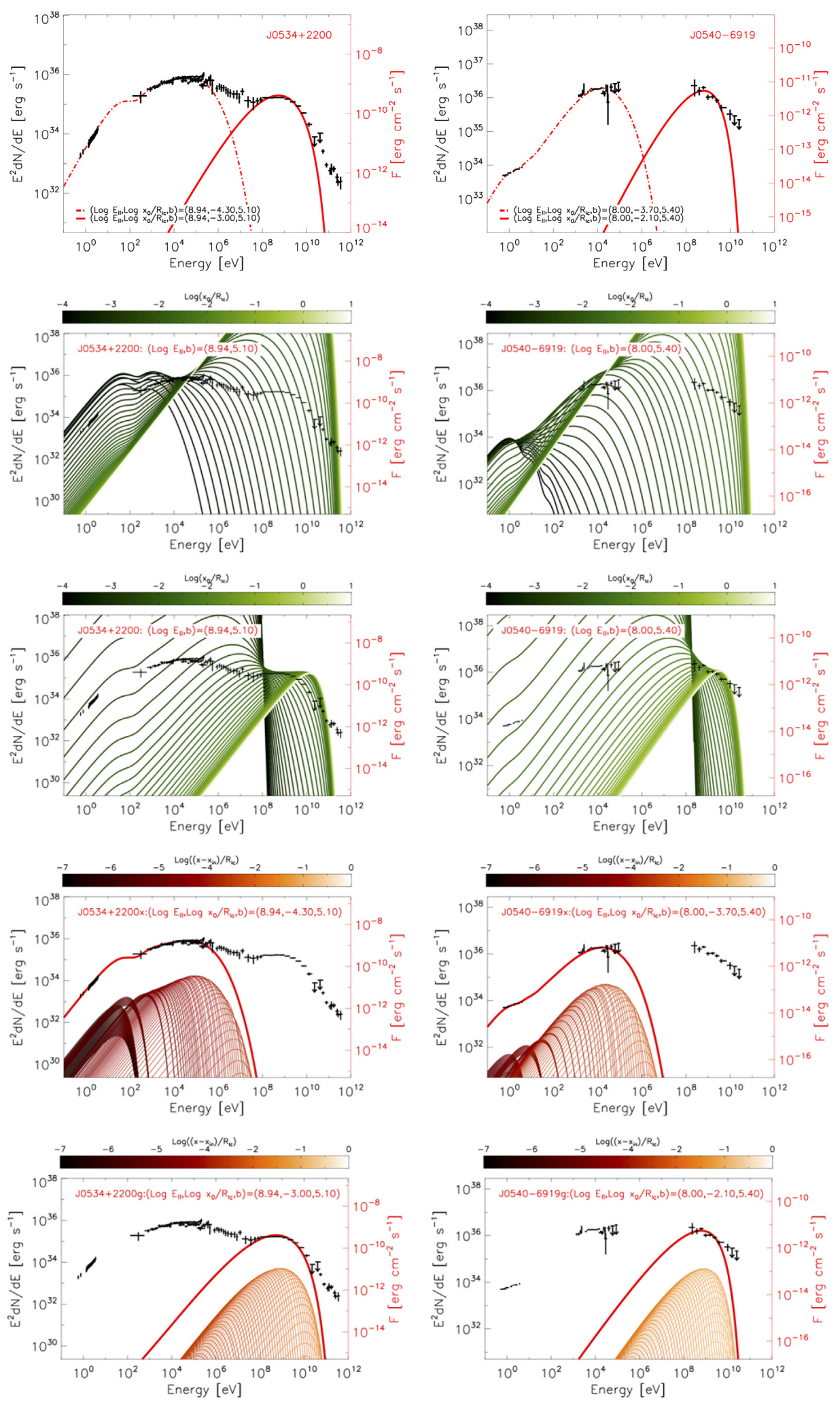

Figure 14. Analysis of the model results for Crab (left-hand panels) and the Crab Twin (right-hand panels), located in the Large Magellanic Cloud. See the text for discussion.

Khangulyan (2012), Lyutikov (2013), Osmanov \& Rieger (2017), and Harding et al. (2018).

Tests regarding the changing of $x_{\text {in }}$ or $x_{\text {out }}$, and the size of the accelerating region were also run to see whether the overall spec- trum from optical to gamma-rays could be better fitted, eventually by just a single set of parameters. We find either these changes were innocuous or that they produced worse spectral representations. Another tests we run regarded the possibility of having an evolution 


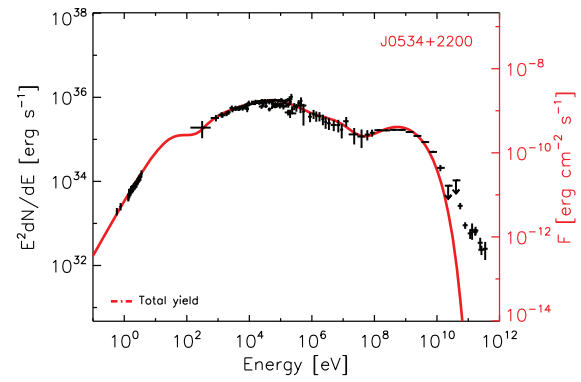

Figure 15. Crab fitting: An additional set of $\left(x_{0}, N_{0}\right)$ is here assumed (for which the values are given in the text) to emphasize that the representation of the particle population might simply be what is behind the $\mathrm{MeV}$ underprediction shown in Fig. 14. For a discussion of the TeV data, see the text.

of $b$ across the accelerating region (from a high to a low value). This is motivated by the fact that a lower value of $b$ assumed when fitting the gamma-ray data only would also produce an SED closer to the $\mathrm{MeV}$ data (the same problems with the underprediction of the $\mathrm{TeV}$ data would be maintained, though). But of course, the value of $b$ that is most influential in the SED is the one valid at the synchrotrondominated part of the spectrum, and once this is fixed, little change is found admitting further variations along the trajectory.

\subsection{Crab twin}

For the Crab twin in the Large Magellanic Cloud only a less constraining data set is available: we use the gamma-ray data from Ackermann et al. (2015) reduced by 25 percent assuming - as these authors say - that about 25 per cent of the flux is contributed by a diffuse environment, as well as the compilation by Kuiper \& Hermsen (2015), and optical to infrared (IR) data from Mignani et al. (2012). The fact that the optical flux density is characterized by a power-law spectrum and the luminosities correlate with the rotational power, like in Crab and other pulsars, prompted Mignani et al. (2012) to suggest that this emission is non-thermal. Given the not-fully understood jump in flux density just recently found in near ultraviolet (NUV), and under the assumption that it might be related with local absorption or an increased continuum contribution due to a non-magnetospheric component, or other effects, see fig. 3 in Mignani et al. (2019) for the data and the associated discussion regarding these issues, we choose not to include the two UV data points in our modelling. We note in any case that only the NUV point is problematic (as it implies a jump of about 1 order of magnitude from adjacent data points in a $v f_{v}$ diagram, what makes it impossible for any model based on a single process with a continuous population of particles). Instead, the far-UV data point in Mignani et al. (2019) would be in agreement with our model, despite we are not fitting against it.

It is important to note that the difference between the gamma-ray data sample of Crab and its twin is significant: the Crab data in gamma-rays have errors smaller than 1 per cent, whereas the errors for the Crab Twin range 10-50 per cent, in addition to the systematic uncertainty mentioned. This will have an impact in the modelling. In fact, when the gamma-ray data set is modelled by itself only a broad range of admissible values of $E_{\|}$are obtained. Because of this, when searching for a separate fit using the same values of $\left(E_{\|}, b\right)$ we shall model first the optical to X-ray data (featuring extremely small error bars) and use the defined values for the pair to see whether a fit to the gamma-ray data is obtained. This is why the gamma-ray fit parameters described in the last panel of Table 2 has no errors. The results of the separate fitting with the same physical parameters of the accelerating region are shown in the right-hand panels of Fig. 14. The similarities with the Crab fitting are striking, and visible along all plots. They are also similar regarding the fitting parameters. Also a high value of $b$ is required for the Crab Twin fit. These values of $b$ are the highest we found when fitting the pulsars with $P>10 \mathrm{~ms}$ in our sample (see Table 1), and promote the possibility that they could relate with the rotational power, or with the magnetic field at the light cylinder.

\section{CONCLUSIONS}

This paper presents a systematic theoretical analysis of the SED of non-thermally emitting pulsars for which the SED is sampled from X-rays (in some cases, also from IR/optical) to gamma-rays. We used the model introduced by Torres (2018), which was found able to describe well the emission of several pulsars using only three physical parameters and a global (normalization) scale. These four parameters clearly distinguish themselves in two groups: the intrinsic ones (the accelerating electric field $E_{\|}$, and the magnetic gradient $b$ ) related to the pulsar itself, and those related to the geometry of the pulsar with respect to the observer (the contrast $\left(x_{0} / R_{\mathrm{lc}}\right)^{-1}$, and the normalization $N_{0}$ for the number of particles flowing along the accelerating region that are emitting radiation towards us). Here, our theoretically scrutinized sample contains 36 of the 40 cases compiled by Coti Zelati et al. (2019), see also Kuiper \& Hermsen (2015) and references therein, and includes 32 normal pulsars and 4 MSPs having broad-band non-thermal SEDs. We have only left out of our analysis a normal pulsar for which only a few data points could be derived in total, making fits less relevant (J2043+2740), and 3 MSPs (B1821-24, B1937+21, and J0218+4232) requiring additional Fermi-LAT observational analysis before fits can proceed. For the latter, though, we have already mentioned preliminary results. This collection of pulsars has to be compared with the original reduced sample of eight sources studies by Torres (2018).

A comparison with such a large data set is a tour-de-force for any model, but particularly for one hosting such a reduced number of free variables. However, we found that for half of the pulsars herein considered (18 out of 36), the model is able to qualitatively describe well the observational data across seven orders of magnitude in energy. This is explicitly shown in Fig. 1. In addition, we have identified a group of eight pulsars for which the model can still be argued to provide the basic features of the SED, although with caveats (see Fig. 5); and a different group of 10 pulsars (including Crab and the Crab twin) for which the model fails - in its original incarnation, i.e. with the three parameters and the normalization scale - to correctly describe the SEDs (see Fig. 6). We studied how this inability happens, realizing of a simple extension of the model that can lead to good fits in all cases: with the same pair of values $\left(E_{\|}, b\right)$ and two different pairs of values $\left(x_{0} / R_{\mathrm{lc}}, N_{0}\right)$, the model can fit well all SEDs, both the X-ray and the gammaray data. This approach separates the model input from related to orientation and geometry from the properties intrinsic to the pulsar, as described above. We have provided two different interpretations (see Fig. 12 and Section 4) that explain why this extension is to be naturally expected. It would be the most probable outcome if two regions of acceleration are visible to the observer and/or when the visibility of one accelerating region is favourable enough so that a more complex representation of the particle distribution is needed. Such model extension, then, does not change any of the model 
basic assumptions at all, and only adds two parameters. Overall, it still makes for an austere model, particularly when the parameters being added are of the same kind and bears full resemblance with the ones previously existing. The fact that with the inclusion of this extension the SEDs for all pulsars analysed can be described with the utmost detail reinforces the conclusions obtained earlier regarding the utility of their free variables as order parameters in the SEDs complexity. As an interesting aside, our interpretation of the SEDs in these cases allows for a qualitative explanation of the $\mathrm{X}$-ray to gamma-ray light-curve phase shifts, what up to now have remained elusive.

We also remarked how well the model can act as a predictor for those pulsars that can be detectable in X-rays or lower energies, if knowing their high energy yield. In all cases where a single set of parameters $\left(E_{\|}, b, x_{0} / R_{\mathrm{lc}}, N_{0}\right)$ will be enough to describe the SED, the matching between the fit to the gamma-ray data only (assumed to be the only data set known) and the fit to the total X-ray and gamma-ray data is surprising. This happens in essentially all the 18 such cases shown in Fig. 1. However, we pointed the caveat that when a single set of parameters is not enough, the gamma-ray yield modelling may miss detectable pulsars, judging them as nondetectable. Luckily, however, this does not act the other way around. The predictive power may thus be incomplete, but if a gamma-ray fit predicts a detectable source, we find there is no risk in searching. Li et al. (2018) have already shown this model can be a successful searching tool for pulsar detections, and more may yet to come with future surveys. With a population of observed non-thermal X-ray pulsars that is currently $>5$ times smaller than that in gamma-rays, such predictive power is welcomed.

The analysis of this larger sample also confirms other previous findings. The appearance of sub-exponential cutoffs in the FermiLAT energy range is, as we advanced in Torres (2018), also a natural consequence of synchrocurvature-dominated losses. We continue to find the great importance that synchrotron and synchrocurvature emission (i.e. the full process without approximations, whether neither synchrotron nor curvature dominates) have for the spectral formation of all the pulsars analysed. We can conclude that fully curvature-dominated emission plays a significant role only in a few cases, but not in most of the gamma-ray emitting pulsars. This is particularly visible in the explicit cases for which we show the pulsar SED formation (see Figs 2, 11, and 14). Such figures are consistent with the properties derived from the solution of the equations of motion (see e.g. Fig. 3). Regions of the trajectory between $\sim 10^{-5}$ and $\sim 10^{-2} R_{\mathrm{lc}}$, where both synchrotron and curvature are relevant, are responsible for most of the observed emission. All in all, we continue to find that the relevant scale for the production of the pulsar's SED are small in comparison with the light cylinder radius.

Whereas for most cases, values of the magnetic gradient around $b=3$ are needed to describe the pulsar SED correctly, we are finding that relative larger magnetic gradients appear in the fits of a few pulsars. In particular, in comparison with the radial dipole magnetic field line value of 3 , we find that for the young and energetic Crab and Crab Twin, and especially for the MSPs, magnetic gradients of up to $b=6$ are needed. This may indicate that the local conditions of the magnetic field where the small accelerating region is located dominates the whole emission. This gradient may be different to the overall behaviour of the field at larger scales, which may continue to be (for instance) a dipole. May the relatively large gradients in these cases, together with the smallness of the relevant emission region, point towards a region of acceleration close to the null point (after which the separatrix goes along the equatorial plane)?
In such region, large numerical resolution works (see fig. 11 of Timokhin 2006, and references therein for earlier theoretical work) have shown that the field is subject to a large variation, where magnetic gradients can be very high.

Another aspect that we find confirmed with our larger sample is the correlation between $E_{\|}$and $x_{0}$. When using the single set fittings together with the separate fits in gamma-rays (i.e. with the $x_{0}$ corresponding to the one obtained in the gamma-ray fit) we see that the generally reduced error bars of our parameters confirm the gamma-ray trend shown in fig. 5 of Torres (2018) using Fermi 2PC data only. Plotting $E_{\|}$versus the $x_{0}$ resulting from the separate Xray fitting the trend is still conserved, displaced to lower values of $x_{0}$. We do not find such clear correlations between direct pulsar properties and model parameters, but only hints in $b$ versus $E_{\|}$, or the spin-down power $E_{\text {sd }}$ versus $E_{\|}$, and the light cylinder field $B_{\mathrm{lc}}$ versus $E_{\|}$, especially for the separate fittings. In particular, we do not see a clear correlation between $b$ and $B_{\mathrm{lc}}$ or $R_{\mathrm{lc}}$ or $B_{\mathrm{s}}$. It is not ruled out that correlations between model and pulsar intrinsic parameters, derived from $(P, \dot{P})$, may appear not one-toone but using combinations of the latter (i.e. with combinations of $B_{\mathrm{lc}}, E_{\mathrm{sd}}$, etc.).

It is interesting to note that there are a few other non-thermal Xray pulsars with no gamma-ray emission yet detected (see Kuiper \& Hermsen 2015, and fig. 2 of Torres 2018). In the context of our findings, these can be a priori produced by a low(er) $E_{\|}$, or by a particular geometrical orientation, where, for instance, only the region of acceleration with the geometry leading to the smaller contrast is visible. However, in Torres (2018) the fits were already done with $E_{||}$varying at least from $10^{4}$ to $10^{10} \mathrm{~V} \mathrm{~m}^{-1}$, and most of the pulsars with no gamma-ray emission were found to prefer a low value of $E_{\|}$with a relatively high value of $x_{0} / R_{\mathrm{lc}}$, instead of a relatively high value of $E_{\|}$with a low value of $x_{0} / R_{\mathrm{lc}}$, as it happens here. This may imply that we are indeed seeing in these pulsars the tail of the population (towards lower $E_{\|}$).

We would like to conclude with a note about Crab and the Crab Twin. Despite the difference in data quality of the data sets for the two pulsars, our analysis unveils some similarities: they are both showcased having large magnetic gradients in the accelerating regions generating the radiation we see. We find that the Crab Twin current data set, and most of the Crab data set can be well described with the model as explained, and have explained how the Crab $\mathrm{MeV}$ energy regime that is missed can actually be encompassed in a more complete (less bumpy) representation of the particle distribution. As an aside, we note that whereas the very high energy data at $\mathrm{TeV}$ energies cannot be encompassed in our model if the acceleration regime has the same vale of $E_{\|}$as the one needed to produce the X-ray and gamma-ray emission, it is possible for it to arise via curvature radiation from a different and more localized magnetospheric extent, without invoking additional processes, such as inverse Compton.

\section{ACKNOWLEDGEMENTS}

DFT and FCZ acknowledge support from the Spanish Ministry of Economy, Industry and Competitiveness grants PGC2018-095512B-I00, SGR2017-1383, and AYA2017- 92402-EXP. DV acknowledges support from the Spanish Ministry of Economy, Industry and Competitiveness grants AYA2016-80289-P and AYA2017-82089ERC (AEI/FEDER, UE). FCZ is supported by a Juan de la Cierva fellowship. DFT acknowledges the participants of the Workshop on Magnetospheres of Neutron Stars and Black Holes (June 2019, Goddard Space Flight Center) for comments. 


\section{REFERENCES}

Abdo A. A. et al., 2013, ApJS, 208, 17

Ackermann M. et al., 2015, Science, 350, 801

Aharonian F. A., Bogovalov S. V., Khangulyan D., 2012, Nature, 482, 507

Aleksić J. et al., 2011, ApJ, 742, 43

Aliu E. et al., 2008, Science, 322, 1221

Aliu E. et al., 2011, Science, 334, 69

Ansoldi S. et al., 2016, A\&A, 585, A133

Bühler R., Blandford R., 2014, Rep. Prog. Phys., 77, 066901

Cheng K. S., Zhang J. L., 1996, ApJ, 463, 271

Coti Zelati F., Torres D. F., Li J., Viganò D., 2019, MNRAS, Submitted

Gotthelf E. V., Bogdanov S., 2017, ApJ, 845, 159

Harding A. K., Kalapotharakos C., Barnard M., Venter C., 2018, ApJ, 869, L18

Kuiper L., Hermsen W., 2015, MNRAS, 449, 3827

Kuiper L., Hermsen W., Cusumano G., Diehl R., Schönfelder V., Strong A., Bennett K., McConnell M. L., 2001, A\&A, 378, 918

Li J., Torres D. F., Coti Zelati F., Papitto A., Kerr M., Rea N., 2018, ApJ, 868, L29

Lyutikov M., 2013, MNRAS, 431, 2580
Marelli M. et al., 2014, ApJ, 790, 51

Mignani R. P., De Luca A., Hummel W., Zajczyk A., Rudak B., Kanbach G., Słowikowska A., 2012, A\&A, 544, A100

Mignani R. P. et al., 2019, ApJ, 871, 246

Osmanov Z., Rieger F. M., 2017, MNRAS, 464, 1347

Sollerman J., Lundqvist P., Lindler D., Chevalier R. A., Fransson C., Gull T. R., Pun C. S. J., Sonneborn G., 2000, ApJ, 537, 861

Timokhin A. N., 2006, MNRAS, 368, 1055

Torres D. F., 2018, Nat. Astron., 2, 247

Tziamtzis A., Lundqvist P., Djupvik A. A., 2009, A\&A, 508, 221

Viganò D., Torres D. F., 2015, MNRAS, 449, 3755

Viganò D., Torres D. F., Hirotani K., Pessah M. E., 2015a, MNRAS, 447 , 1164

Viganò D., Torres D. F., Hirotani K., Pessah M. E., 2015b, MNRAS, 447, 2631

Viganò D., Torres D. F., Hirotani K., Pessah M. E., 2015c, MNRAS, 447, 2649

Viganò D., Torres D. F., Martín J., 2015d, MNRAS, 453, 2599

This paper has been typeset from a $\mathrm{T}_{\mathrm{E}} \mathrm{X} / \mathrm{L} \mathrm{T} \mathrm{E} \mathrm{X}$ file prepared by the author. 